\title{
LIMIT THEOREMS FOR SOLUTIONS OF STOCHASTIC DIFFERENTIAL EQUATION PROBLEMS
}

\author{
J. VOM SCHEIDT and W. PURKERT \\ Ingenieurhochschule Zwickau \\ DDR-95 Zwickau \\ Germany \\ (Received September 5, 1978)
}

ABSTRACT. In this paper linear differential equations with random processes as coefficients and as inhomogeneous term are regarded. Iimit theorems are proved for the solutions of these equations if the random processes are weakly correlated processes.

Limit theorems are proved for the eigenvalues and the eigenfunctions of eigenvalue problems and for the solutions of boundary value problems and initial value problems. KEY WORDS AND PHRASES. Limit Theorem, Stochastic Eigenvalue Problem, Stochastic Boundary Value Problem, Differential Equation. 1980 MATHEMATICS SUBJECT CLASSIFICATION CODES. 60, 60H. 
1.. IIMTRODUCTION.

At the research of physical and engineering problems it is of great importance the approach to the differential equations with atochastic proceswes as coefficients respectively with otochastic boundary or initial conditions. There is a series of papers which deal with such a problem. The first moments of the solution are often calculated from the first moments of the stochastic processes involving the problem. For the applications this is an interesting and important problem (see [4]). The calculation of the distributions of the salution processes from the distributions of the involving processes is often more difficult. This problem contains already many difficulties for very aimple problems (e.g. for the initial value problem of a linear ordinary differential equation of the first order with atochastic coefficients). If one specializes the stochastic processes involving that problem then one succeeds in a few cases in obtaining statements on the di atribution of the solution. As an example of such a result we refer to the paper of G.E. Uhlenbeck und I.S. Ornatein [8]. They regard such stochastic inputs which do not possess a "distant effect", i.e. the values of the process do not possess a correlation if the distance between the observation points is Iarge. As a result they can show that the solution of a special initial value problem with such a process without "distant effect" as the right side is approximativly a so-called Ornstein-Uhlenbeck-process, i.e. a process for which the first distribution function is a Gaussian distribution function.

These processes without "distant effect" were defined exactly by the authors in the paper [6] through the process class of the "weakly correlated processes" and they were applied in this paper at the consideration of stochastic eigenvalue problems and boundary value prob- 
lems. A limit theorem is obtained for the eigenvalues, eigenfunctions of etochastic eigenvalue problems respectively for the solutions of stochastic boundary problems with weakly correlated coefficients. This Iimit theorem shows the approximate Gaussian distribution of the first distribution function of the solutions of eigenvalue problems and boundary value problems.

In the present paper the conception of the weakly correlated process is defined more generally than in the paper [6] (i.e. the stationarity of the process falls out the supposition). The correlation Iength $\varepsilon$ denotes the minimom dietance between observation points of a weakly correlated process that the values of the process do not affect in observation points which possess a distance larger than $\varepsilon$.

In section 2 a few theorems will be proved about fanctionals of wakly correlated proceses which are important for the applications at eigenvalue problems, boundary value problems and initial value problems in the following sections.

Section 3 deals with stochastic eigenvalue problems for ordinary differential equations with deterministic boundary conditions where the coefficient of the differential operator are independent, weakly correlated processes of the correlation length $\varepsilon$. We prove that the eigenvalues and the eigenfunctions as $2+0$ possess a Gaussian distribution. For instance the eigenfunctions of the stochastic eigenvalue problem converge in the distribution as $\varepsilon+0$ to Gaussian processes. Methods of the perturbation theory are essentialiy used. In a general example it is referred to a few remarkable appearances.

In section 4 we deai with stochastic boundary problems and we obtain similar results as for stochastic eigenvalue problems. The case of a Sturm-Liouville-operator with a stochastic inhomogeneous term 
(weakly correlated) which was dealt with by W.E. Boyce in [1] is included in the result of this section. Some limitations relative to the amallness of the atochastic coefficients of the operator but not of the inhomogeneous term are assumed as at the eigenvalue problems, too. We show a calculation of the correlation function by the Ritz-method to eliminate the Green function of the averaged problem from the correlation function of the Iimit process of the ralution of the Boundary ralue problem.

At last, section 5 deals with stochastic initial value problems of ordinary differential equations. The inhomogeneous terms are weakly correlated processes. The results in this theory of the weakly correlated processes as $\varepsilon \downarrow 0$ resemble the results of the Itó-theory if the inhomogeneous terms are replaced by Gaussian white noise according to the Itó-theory and the formed Ito-equation is solved according to the It6-theory. The practice by the help of the weakly correlated processes effers prineipally from the It6-theory. One obtains the Iimit theorems by use of the weakly correlated processes if at first a formala for the a.s. continuous differentiable sample functions of the solutions is derired $(\varepsilon \neq 0)$ and then we go to the Iimit $(\varepsilon \downarrow 0)$. With it we get an approxination of the solution of the initial value problem with weakly correlated processes $(\varepsilon \ll 1, \varepsilon \neq 0)$. In the It6-theory one goes to the limit in the differential equation and this equation is solved by a well worked out mathematical theory. We get different results at this different practice in problems of differential equations in which the coefficients are weakly correlated processes and not the inhomogeneous term. We do not deal with such probIems in this: paper.

It is principaly no distinction in the proof of limit theorems 
for the initial value problems and boundary ralue respectively eigenvalue problems with weakly correlated processes. A widening of the Ito-theory on boundary value respectively eigenvalue problems with white noise for the coefficients seems to contain a few fundamental difficulties.

\section{WEAKLY CORRELATED PROCESSES}

DEFINITION 1. Let $\left(x_{1}, x_{2}, \ldots, x_{n}\right)$ be a finite set of real numbers and $\varepsilon>0$. A subset $\left(x_{i_{1}}, x_{i_{2}}, \ldots, x_{i_{k}}\right) \leqslant\left(x_{1}, x_{2}, \ldots, x_{n}\right)$ is called $\varepsilon-a d-$ joining if

$$
\left|x_{r_{1}}{ }^{-x} r_{2}\right| \leqslant \varepsilon,\left|x_{r_{2}}{ }^{-x} r_{3}\right| \leqslant \varepsilon, \ldots,\left|x_{r_{k-1}}{ }^{-x} r_{k}\right| \leqslant \varepsilon
$$

is fulfilled for the $x_{i}, j=1, \ldots, k$, which are arranged after the quality (these we have termed as $x_{r_{1}}, x_{r_{2}}, \ldots, x_{r_{k}}$ ). A subset of one number is always called $\varepsilon$-adjoining. A subset $\left(x_{i_{1}}, \ldots, x_{i_{k}}\right) \subseteq$ $\left(x_{1}, \ldots, x_{n}\right)$ is called maximum $\varepsilon$-adjoining (relative to $\left(x_{1}, \ldots, x_{n}\right)$ ) if it is $\varepsilon$-adjoining but the subset $\left(x_{i_{1}}, \ldots, x_{i_{k}}, x_{r}\right)$ is not $\varepsilon$-adjoining for $x_{r} \in\left(x_{1}, \ldots, x_{r}\right) \backslash\left(x_{i_{1}}, \ldots, x_{i_{k}}\right)$.

Every finite set $\left(x_{1}, \ldots, x_{n}\right)$ split unique in disjoint maximum c-adjoining subsets.

DEFINITION 2. A stochastic process $f(x, w)$ with $\langle f(x, w)\rangle \dot{\dot{x}}$ $E\{f(x, w)\}=0$ is called weakly correlated of the correlation length 6 when the relation

$$
\begin{aligned}
\left\langle f\left(x_{1}\right) \ldots f\left(x_{n}\right)\right\rangle=\left\langle f\left(x_{11}\right) \ldots f\left(x_{1 p_{1}}\right)\right\rangle\left\langle f\left(x_{21}\right) \ldots f\left(x_{2 p_{2}}\right)\right\rangle \cdot \ldots \\
\cdot\left\langle f\left(x_{k 1}\right) \ldots f\left(x_{k p_{k}}\right)\right\rangle
\end{aligned}
$$

is atisfied for the nth moments (for am $n \geq 1$ ) if the set $\left(x_{1}, \ldots, x_{n}\right)$ splits in the maximum -adjoining subsets $\left(\sum_{i=1}^{k} p_{i}=n\right)$
$\left\{\left(x_{11}, \ldots, x_{1 p_{1}}\right),\left(x_{21}, \ldots, x_{2 p_{2}}\right), \ldots,\left(x_{k 1}, \ldots, x_{k p_{k}}\right)\right\}$

If the process $f(x, \omega)$ is weakly correlated with the correlation Iength $\varepsilon$, then we get for its correlation function 


$$
\langle f(x) f(y)\rangle=\left\{\begin{array}{cl}
R_{\varepsilon}(x, y) & \text { for } y \in K_{\varepsilon}(x) \\
0 & \text { for } y \notin K_{\varepsilon}(x)
\end{array}\right.
$$

where $K_{\varepsilon}(x) \dot{=}\left\{y \in R^{1}:|x-y| \leq \varepsilon\right\}$.

The existence of especially stationary weakly correlated processes has been proved in the paper [6]. In this paper it is also proved that weakly correlated processes with smooth sample functions exist.

In the following a few theorems will be proved about weakly correlated processes. These theorems will be used essentially in the applications at equations of the mathematical physics.

THEOREM 1. Lot $f_{\varepsilon}(x, w)$ be a sequence of weakly correlated processes as $\varepsilon \downarrow 0$ with the correlation functions

$$
\left\langle f_{\varepsilon}(x) f_{\varepsilon}(y)\right\rangle=\left\{\begin{array}{cc}
R_{\varepsilon}(x, y) & \text { for } y \in K_{\varepsilon}(x) \\
0 & \text { for } y \notin K_{\varepsilon}(x)
\end{array}\right.
$$

where is fulfilled

$$
\lim _{\varepsilon+0} \frac{1}{\varepsilon} \int_{-\varepsilon}^{\varepsilon} R_{\varepsilon}(x, x+y) d y \dot{I} a(x) \quad \text { and } a(x) \not \equiv 0
$$

uniformly in $x$. Further on let $g_{j}(x, y), j=1,2$, be in $\left[a-\eta, b_{i}+\eta\right]$ differentiable functions relative to $x\left(\eta>0, b_{i}>a\right)$ and

$$
\sup _{i, x, y}\left\{\left|g_{i}(x, y)\right|,\left|\frac{\partial}{\sqrt{x}} g_{i}(x, y)\right|\right\} \leq c .
$$

When $G_{2}=\left\{\left(x_{1}, x_{2}\right):\left|x_{1}-x_{2}\right| \leqslant \varepsilon, a \leq x_{i} \leq b_{i}\right\}$ then we get for

the relation

$$
r_{i \varepsilon}(x, w)=\frac{1}{\sqrt{\varepsilon}} \int_{a}^{b} f_{\varepsilon}(y, w) g_{i}(y, x) d y
$$

$$
\operatorname{Iim}_{\varepsilon \downarrow 0}\left\langle r_{1 \varepsilon}\left(x_{1}\right) r_{2 \varepsilon}\left(x_{2}\right)\right\rangle=\int_{a}^{\min \left(b_{1}, b_{2}\right)} a(y) g_{1}\left(y, x_{1}\right) g_{2}\left(y, x_{2}\right) d y \text {. }
$$

PROOF. We can set $b_{1} \leqslant b_{2}$ and $\varepsilon<b_{1}$. We substitute in

$$
\begin{aligned}
I_{12} \dot{\dot{j}}\left\langle r_{1 \varepsilon}\left(x_{1}\right) r_{2 \varepsilon}\left(x_{2}\right)\right\rangle & =\frac{1}{\varepsilon} \int_{a}^{b} \int_{a}^{b}\left\langle f_{\varepsilon}\left(y_{1}\right) f_{\varepsilon}\left(y_{2}\right)\right\rangle g_{1}\left(y_{1}, x_{1}\right) g_{2}\left(y_{2}, x_{2}\right) d y_{1} d y_{2} \\
& =\frac{1}{\varepsilon} \iint_{G_{2}} R_{\varepsilon}\left(y_{1}, y_{2}\right) g_{1}\left(y_{1}, x_{1}\right) g_{2}\left(y_{2}, x_{2}\right) d y_{1} d y_{2}
\end{aligned}
$$

$z_{1}=y_{1}, z_{2}=y_{2}-y_{1}$ and obtain (see Fig.1) 


$$
I_{12}=\frac{1}{\varepsilon}\left[\iint_{Q} h\left(z_{1}, z_{2}\right) d z_{1} d z_{2}-\iint_{Q_{1}} h\left(z_{1}, z_{2}\right) d z_{1} d z_{2}\right]
$$

with $h\left(z_{1}, z_{2}\right)=R_{E}\left(z_{1}, z_{1}+z_{2}\right) g_{1}\left(z_{1}, x_{1}\right)_{g_{2}}\left(z_{1}+z_{2}, x_{2}\right)$

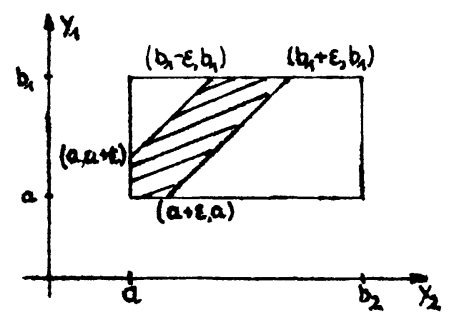

Fig.1

and $Q=\left\{\left(z_{1}, z_{2}\right): a \leqslant z_{1} \leqslant b_{1},-\varepsilon \leqslant z_{2} \leq \varepsilon\right\}$. Ay $\operatorname{Iim}_{\varepsilon \downarrow 0} \frac{1}{\varepsilon} \iint_{Q_{1}} d z_{1} d z_{2}=0$ and $\left|h\left(z_{1}, z_{2}\right)\right| \leqslant\left(R_{\varepsilon}\left(z_{1}, z_{1}\right) R_{\varepsilon}\left(z_{1}+z_{2}, z_{1}+z_{2}\right)\right)^{1 / 2} C^{2}$ it follows

$$
\operatorname{Iim}_{\varepsilon \downarrow 0} I_{12}=\operatorname{Iim}_{\varepsilon \neq 0} \frac{1}{\varepsilon} \int_{a}^{b_{1}} g_{1}\left(z_{1}, x_{1}\right) \int_{-\varepsilon}^{\varepsilon} R_{\varepsilon}\left(z_{1}, z_{1}+z_{2}\right) g_{2}\left(z_{1}+z_{2}, x_{2}\right) d z_{2} d z_{1} \text {. }
$$

At last we obtain

$$
\begin{aligned}
\lim _{\varepsilon \downarrow 0} I_{12} & =\lim _{\varepsilon \downarrow 0} \int_{a}^{b_{1}} g_{1}\left(z_{1}, x_{1}\right) \frac{1}{\varepsilon} \int_{-\varepsilon}^{\varepsilon} R_{\varepsilon}\left(z_{1}, z_{1}+z_{2}\right)\left\{z_{2}\left(z_{1}, x_{2}\right)+o\left(z_{2}\right)\right\} d z_{2} d z_{1} \\
& =\int_{a}^{b_{1}} g_{1}\left(z_{1}, x_{1}\right) g_{2}\left(z_{1}, x_{2}\right) \lim _{\varepsilon \downarrow 0} \frac{1}{\varepsilon} \int_{-\varepsilon}^{\varepsilon} R_{\varepsilon}\left(z_{1}, z_{1}+z_{2}\right) d z_{2} d z_{1}
\end{aligned}
$$

if the function $g_{2}\left(z_{1}+z_{2}, x_{2}\right)$ is developed relative to $z_{2}$ at $z_{2}=0$. The theoren is proved.

Before we denote the more important theorem 3, we prove a simple theorem.

THEORRM 2. It is $m_{n}=\left\{\left(x_{1}, \ldots, x_{n}\right): a \leqslant x_{i} \leqslant b_{i}, i=1,2, \ldots, n\right\}$ and $G_{n}=$ $\left\{\left(x_{1}, \ldots, x_{n}\right) \in \mathbb{W}_{n}:\left(x_{1}, \ldots, x_{n}\right) \quad \varepsilon\right.$-adjoining $\}$. Let $g\left(x_{1}, \ldots, x_{n}\right)$ be a function which is in $\left\{\left(x_{1}, \ldots, x_{n}\right): a-\eta \leqslant x_{i} \leqslant b_{i}+\eta, i=1, \ldots, n\right\}$ limited: $\left|g\left(x_{1}, \ldots, x_{n}\right)\right| \leq c$. Then the integral $\int_{G_{n}} g\left(x_{1}, \ldots, x_{n}\right) d x_{1} \ldots d x_{n}$ is at least of the order $\varepsilon^{n-1}$ for $\varepsilon \downarrow 0$.

PROOF. By $\bar{b}=\min \left(b_{1}, b_{2}, \ldots, b_{n}\right)$ it is

$$
\left|\int_{G_{n}} g\left(x_{1}, \ldots, x_{n}\right) d x_{1} \ldots d x_{n}\right| \leqslant C n ! \int_{a}^{b} d x_{1} \int_{x_{1}}^{x_{1}+\varepsilon} d x_{2} \ldots \int_{x_{n-1}}^{x_{n-1}+\varepsilon} d x_{n}=C n ! \varepsilon^{n-1}(\bar{b}-a)
$$

where the term $\int_{a}^{\delta} d x_{1} \int_{x_{1}}^{x_{1}+\varepsilon} d x_{2} \ldots \int_{x_{n-1}}^{x_{n-1}+\varepsilon} d x_{n}$ denotes the volume of 


$$
H_{\varepsilon}^{1} \cdots n=\left\{\left(x_{1}, \ldots, x_{n}\right): a \leqslant x_{1} \leqslant 5, x_{1} \quad x_{2} \leqslant x_{1}+\varepsilon, \ldots, x_{n-1} \leqslant x_{n} \leqslant x_{n-1}+\varepsilon\right\}
$$

and we get $G_{\varepsilon}^{1} \ldots n_{\leqslant} H_{\varepsilon}^{1 \ldots} \ldots n . G_{\varepsilon}^{i} 1 \ldots i_{n}$ marks the set of the $\varepsilon$-adjoining points in $G_{n}$ with $x_{i_{1}} \leqslant x_{i_{2}} \leq \ldots \leq x_{i_{n}}$. Then the above giren inequality results from

$$
G_{n}=\bigcup_{r \in \sigma(1, \ldots, n), \pi=\left(\begin{array}{l}
1 \\
i_{1} \ldots i_{n}
\end{array}\right)} G_{\varepsilon}^{i_{1} \cdots i_{n}}
$$

and with it the statement of the theorem:

THEORM 3. Let $f_{\varepsilon}(x, \omega)$ be a sequence of weakly correlated processes as $\varepsilon \downarrow 0$. The absolute moments $\left\langle\left|f_{\varepsilon}(x, w)\right| j\right\rangle=c_{j}(x)$ are to exist and $c_{j}(x) \leqslant c_{j}$. Let $g_{i}(x, y), i=1,2, \ldots, n$, be in $\left[a-\eta, b_{i}+\eta\right]$ differentiable functions relativ to $x\left(\eta>0, b_{i}>a\right)$ and

Then we have for

$$
\sup _{i, x, y}\left\{g_{i}(x, y),\left|\frac{\partial}{\partial x} g_{i}(x, y)\right|\right\} \leqslant c .
$$

the relation

$$
r_{i \varepsilon}(x, w)=\frac{1}{\sqrt{\varepsilon}} \int_{a}^{b} f_{\varepsilon}(y, w) g_{i}(y, x) d y
$$$$
\begin{aligned}
& \operatorname{Iim}_{\varepsilon \downarrow 0}\left\langle r_{1 \varepsilon}\left(x_{1}\right) r_{2 \varepsilon}\left(x_{2}\right) \ldots r_{n \varepsilon}\left(x_{n}\right)\right\rangle= \\
& =\left\{\begin{array}{cc}
\sum_{\left(i_{1}, i_{2}\right), \ldots,\left(i_{n-1}, i_{n}\right)} \operatorname{Iim}_{\varepsilon \downarrow 0}^{i m}\left\langle r_{i_{1} \varepsilon} r_{i_{2} \varepsilon}\right\rangle \lim _{\varepsilon \downarrow 0}\left\langle r_{i_{3} \varepsilon} r_{i_{4}}\right) \ldots \lim _{\varepsilon \downarrow 0}\left\langle r_{i_{n-1} \varepsilon} r_{i_{n} \varepsilon}\right\rangle \\
0 & \text { for } n \text { even }
\end{array}\right.
\end{aligned}
$$

The sum extends for all splittings of $(1, \ldots, n)$ in pairs $\left(i_{1}, i_{2}\right)$, $\ldots,\left(i_{n-1}, i_{n}\right)$ with $i_{r}<i_{r+1}$ for every pair $\left(i_{r}, i_{r+1}\right)$. Splittings are equal which differ through the sequence of the pairs.

The proof of this theorem submits similar to the proof of theorem 7 in [6] by the help of the above given theorem 1 and it also submits the proof of the following theorem 4 from the proof of the theorem 8 in [6].

THEOREM 4. Let $f_{1 \varepsilon}(x, w), \ldots, f_{n \varepsilon}(x, w)$ be sequences of independent weakly correlated processes as $\varepsilon \nmid 0$. Let $g_{i j}(x, y), i=1, \ldots, k, j=1, \ldots, n$, be in $\left[a-\eta, b_{i}+\eta\right], i=1, \ldots, k$, differentiable functions relativ to $x$ and 


$$
\sup _{i, j, x, y}\left\{\left|g_{i j}(x, y)\right|,\left|\frac{\partial}{\partial x} g_{i j}(x, y)\right|\right\} \leqslant c .
$$

Then we have for

$$
r_{i \varepsilon}^{(j)}(x, w)=\frac{1}{\sqrt{\varepsilon}} \int_{a}^{b} f_{j}(y, w) g_{i j}(y, x) d y
$$

the relation $\left(r_{i \varepsilon}^{(j)}\left(x_{i}, \omega\right) \stackrel{\dot{\prime}}{=} r_{i \varepsilon}^{(j)}\right)$

$$
\lim _{\varepsilon \downarrow 0}\left\langle\left(r_{1 \varepsilon}^{(1)}+\ldots+r_{1 \varepsilon}^{(n)}\right) \ldots\left(r_{k \varepsilon}^{(1)}+\ldots+r_{k \varepsilon}^{(n)}\right)\right\rangle= \begin{cases}\text { rim } A_{\varepsilon} \text { for } n \text { even } \\ 0 & \text { for } n \text { odd }\end{cases}
$$

with

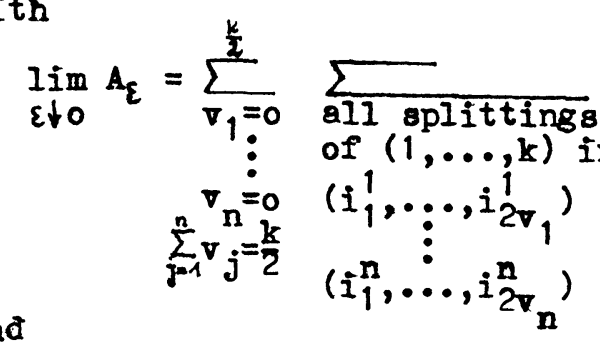

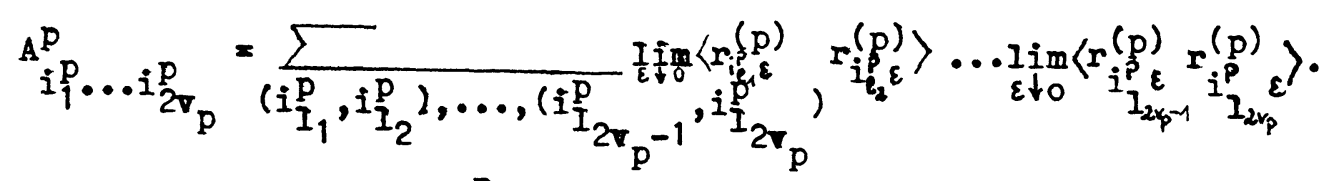

The sum in the term for $A_{i}^{p} \ldots i_{2 \nabla}^{p}$ extends for all splittings of $\left(i \frac{p}{p}, \ldots, i \frac{p}{2 r_{p}}\right)$ in pairs like in theorem 3 .

As an application of the theorems 1 to 4 we will prove the theoren 5.

THEOREM 5. Let $f_{1 \varepsilon}(x, \omega), \ldots, f_{n \varepsilon}(x, w)$ be sequences of independent, weakly correlated processes with continuous sample functions as the correlation length $\varepsilon \downarrow 0$. Let $g_{i j}(x, y)$ be differentiable functions in $[a-\eta, b+\eta] \times[a, b]$ relative to $x$ with the conditions of the limited Iike in theorem 4. Then the stochastic vector process

$$
\begin{aligned}
& \left.\underline{r}_{\varepsilon}(x, \omega)=\left(r_{i \varepsilon}(x, w)\right)_{1 \leqslant i \leqslant m}^{T} 1\right) \\
& \text { with } r_{i \varepsilon}(x, w)=\frac{1}{\sqrt{\varepsilon}} \sum_{j=1}^{n} \int_{a}^{F} g_{i j}(y, x) P_{j \varepsilon}(y, w) d y
\end{aligned}
$$

converges in the distribution as $\varepsilon+0$ to a Gaussian vector process

1) Matrices are denoted by underlining. 
$\xi(x, \omega)=\left(\xi_{i}(x, w)\right)_{1}^{T} \leqslant i \leftarrow m$ with $\xi_{i}(x, w)=\sum_{j=1}^{n} \xi_{i j}(x, w)$ where the Gaussian processes $\underline{\xi}^{j}(x, w)=\left(\xi_{i j}(x, w)\right)_{1 \leqslant i \leqslant m}^{T}, j=1, \ldots, n$, are independent and we have $\left\langle\mathbf{y}^{j}(x)\right\rangle=0, \quad \min \left(x_{1}, x_{2}\right)$

$\left\langle\sum^{j}\left(x_{1}\right) j^{j}\left(x_{2}\right)^{T}\right\rangle=\left(\int_{a} a_{j}(y)_{g_{p j}}\left(y, x_{1}\right)_{g_{q j}}\left(y, x_{2}\right) d y\right)_{1 \leqslant p, q \leqslant m^{\prime}}$ i.e. all the distribution functions of $\underline{r}_{\varepsilon}(x, w)$ converge a $\varepsilon+0$ to the adequate distribution functions of the Gaussian vector process $\xi(x, w)$. It is for the weakly correlated processes $f_{i \varepsilon}(x, w), i=1, \ldots, n$,

$$
\left\langle f_{i \varepsilon}(x) f_{i \varepsilon}(y)\right\rangle=\left\{\begin{array}{cc}
R_{i \varepsilon}(x, y) & \text { for } y \in K_{\varepsilon}(x) \\
0 & \text { for } y \notin K_{\varepsilon}(x)
\end{array}\right.
$$

and $a_{i}(x) \doteq \lim _{\varepsilon \neq 0} \frac{1}{\varepsilon} \int_{-\varepsilon}^{\varepsilon} B_{i \varepsilon}(x, x+y) d y \quad\left(a_{i} \neq 0\right)$.

PROOF. At first we calculate the limit of the $k$-th moment with

the theoreme 3 and 4

$$
\left\langle r_{a_{1} \varepsilon}\left(x_{1}\right) x_{a_{2} \varepsilon}\left(x_{2}\right) \ldots r_{a_{k} \varepsilon}\left(x_{k}\right)\right\rangle \quad \text { with } a_{p} \in\{1,2, \ldots, m\}, x_{p} \in[a, b]
$$

and we obtain by $r_{i \varepsilon}(x, \omega)=\sum_{j=1}^{n} r_{i j \varepsilon}(x, \omega)$,

then

$$
r_{I j \varepsilon}(x, \omega)=\frac{1}{\varepsilon} \int_{a}^{x} g_{i j}(y, x) f_{j \varepsilon}(y, w) d y
$$

$$
\begin{aligned}
& \operatorname{Iim}_{\varepsilon+0}\left\langle r_{a_{1} \varepsilon}\left(x_{1}\right) \ldots r_{a_{k} \varepsilon}\left(x_{k}\right)\right\rangle=\operatorname{Iim}_{\varepsilon+0}\left\langle\left(\sum_{j=1}^{n} r_{a_{1} j \varepsilon}\left(x_{1}\right)\right) \ldots\left(\sum_{j=1}^{n} r_{a_{k} j \varepsilon}\left(x_{k}\right)\right)\right\rangle \\
& \begin{array}{ll}
\sum_{\nabla_{1}=0} & \sum_{\text {all splittings }} \\
\vdots & \text { of }(1, \ldots, k) \text { in } \\
\sum_{j=1}^{n} \nabla_{n}=0 & \left(i_{1}^{1}, \ldots, \ldots, i_{2 v_{1}}\right)
\end{array}
\end{aligned}
$$

It is

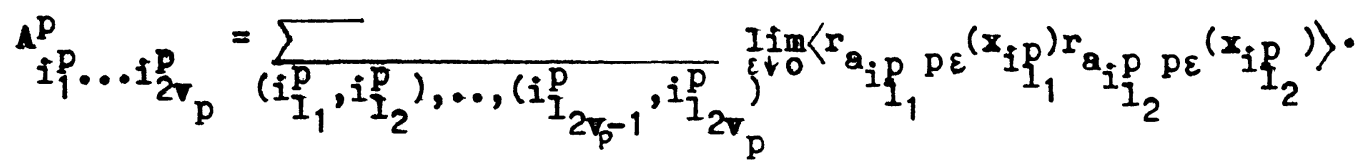

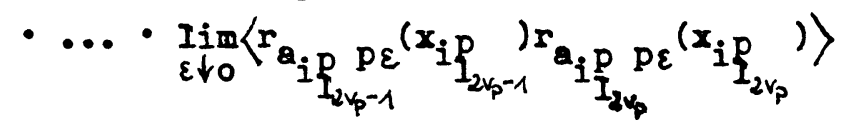

and from theorem 1 


$$
\lim _{i \text { o }}\left\langle r_{a_{i} p \varepsilon}\left(x_{i}\right) r_{a_{j} p \varepsilon}\left(x_{j}\right)\right\rangle=\int_{a}^{\min \left(x_{i}, x_{j}\right)} a_{p}(y) g_{a_{i} p}\left(y, x_{i}\right) g_{a_{j} p}\left(y, x_{j}\right) d y \text {. }
$$

We introduce the Gaussian process $g^{p}(x, \omega)=\left(\xi_{i p}(x, \omega)\right)_{1 \leqslant i \leqslant m}^{T}$ with the eoments $\left\langle\epsilon^{p}(x)\right\rangle=0$,

and obtain

$$
\left.\left\langle i^{p}\left(x_{1}\right) s^{p}\left(x_{2}\right)^{T}\right\rangle=e_{a}^{\min \left(x_{1}, x_{2}\right)} \int_{p}(y) g_{i p}\left(y, x_{1}\right) g_{j p}\left(y, x_{2}\right) d y\right)_{1 \leqslant i, j k m}
$$

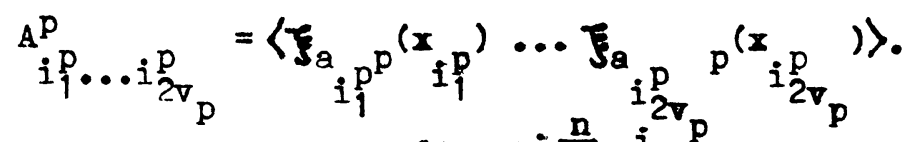

Further on it follows by $\mathbf{L}(x, \omega)=\sum_{j=1}^{n} j^{j}(x, w)$ if the processes $I^{1}(x, \omega), \ldots, \Omega^{n}(x, \omega)$ are supposed independent from $(2)$ with $\boldsymbol{X}(x, \omega)=$ $\left(\xi_{i}(x, \omega)\right)_{1 \leqslant i \leqslant m}^{T}$ the formula

$$
\lim _{\text {sto }}\left\langle r_{a_{1} \varepsilon}\left(x_{1}\right) \ldots r_{a_{k} \varepsilon}\left(x_{k}\right)\right\rangle=\left\langle g_{a_{1}}\left(x_{1}\right) \ldots g_{a_{k}}\left(x_{k}\right)\right\rangle .
$$

Then from (3) we obtain

$$
\lim _{c t o} F_{t x_{1} \ldots x_{8}}\left(\underline{t}_{1}, \ldots, \underline{t}_{8}\right)=\phi_{x_{1} \ldots x_{8}}\left(\underline{t}_{1}, \ldots, t_{8}\right)
$$

with $F_{\varepsilon x_{1} \ldots x_{g}}\left(\underline{t}_{1}, \ldots, \underline{t}_{8}\right)=P\left(\underline{r}_{\varepsilon}\left(x_{q}\right)<\underline{t}_{1}, \ldots, \underline{r}_{\varepsilon}\left(x_{g}\right)<\underline{t}_{8}\right)$ and $\phi_{x_{1} \ldots x_{s}}\left(\underline{t}_{\gamma}, \ldots, t_{s}\right)$ denotes the distribution function of the Gausaian vector $\left(g\left(x_{1}\right), \ldots, s\left(x_{s}\right)\right)$. The theorem 5 is proved.

\section{STOCHASTIC EIGENVAIUE PROBLFMS}

3.1. We regard the stochastic eigenvalue problew

$L(\omega) u \neq(-1)^{m}\left[f_{m}(x) u^{(m)}\right]^{(m)}+\sum_{r=0}^{m-1}(-1)^{r}\left[f_{r}(x, w) u^{(r)}\right]^{(r)}=\lambda u$

$$
u^{(k)}(0)=u^{(k)}(1)=0, k=0,1, \ldots, m-1 \text {. }
$$

Let $\bar{f}_{r}(x, w) \dot{f}_{r}(x, w)-\left\langle f_{r}(x)\right\rangle$ for $0 \leqslant r \leqslant m-1$ be independent, weakly correlated proceases with the correlation length $\varepsilon$ and $a_{.5}$. all the trajectories of $\mathrm{P}_{\mathrm{r}}(x, \omega)$ be continuous. The deterministic runction $f_{m}(x) \neq 0$ has continuous m-th order derivatives. Further on $\left|F_{r}^{(s)}(x, w)\right|<\eta$ is assumed to be with a smaII $\eta$ for $s=0,1, \ldots, r$ and $r=0,1, \ldots, m-1$. The functions $c_{r}(x) \pm\left\langle f_{r}(x)\right\rangle, r=0,1, \ldots, m-1$, possess such properties that the averaged problem to (4) (see [4]) 
124

J.V. SCHELDT AND W. PURKERT

$$
\begin{aligned}
& \langle L(w)\rangle w \dot{ }(-1)^{m}\left[f_{m}(x) w{ }^{(m)}\right]^{(m)}+\sum_{r=0}^{m-1}(-1)^{r}\left[c_{r}(x) w(r)\right]^{(r)}=\mu w \\
& w^{(k)}(0)=w^{(k)}(1)=0, k=0,1, \ldots, m-1
\end{aligned}
$$

is positive definite. Then $L(\omega)$ is also ass. positive definite for $\operatorname{smaIl} \eta \cdot$

Let $\lambda_{1}(w)$ be the eigenvalues of Eq. (4). Further on let $\mu_{1}$ the eigenvalues and $w_{I}(x)$ the eigenfunction of the averaged problem. We assume that the eigenvalues $h_{1}$ are simple. Then we have the development of $\lambda_{1}(w)$

$$
\lambda_{I}(\omega)=\mu_{I}+\lambda_{1 I}(\omega)+\lambda_{2 I}(\omega)+\ldots \text { (convergence } \text { ass. }_{\bullet} \text { ) }
$$

with $\lambda_{1 I}(w)=b_{I I}(w)$ and of the eigenfunction of Eq. (4)

$$
u_{I}(x, w)=w_{I}(x)+u_{1 I}(x, w)+u_{2 I}(x, w)+\ldots
$$

(convergence in $\mathrm{I}_{2}(0,1)$ a.8.) with

$$
u_{1 I}(x, w)=-\sum_{i \neq I}^{\infty} \frac{1}{R_{i I}} b_{i I}(w) w_{i}(x)
$$

(see [4]). It is $\mu_{i j} \dot{\equiv} \mu_{i}-\mu_{j}$ and $b_{i j}(\omega)=\left(I_{1}(\omega) w_{i}, w_{j}\right)$ where

$$
L_{1}(w) u \stackrel{\doteq}{\doteq} L(\omega) u-\langle L\rangle u=\sum_{r=0}^{m-1}(-1)^{r}\left[\bar{f}_{r}(x, v) u^{(r)}\right]^{(r)} \text {. }
$$

The following important theorem 6 is proved in the paper [6].

THEOREM 6. We get for the terms $u_{k I}(x, \omega)$ and $\lambda_{k I}(\omega)$ of the developments of $u_{1}(x, w)$ and $\lambda_{1}(w)$

$$
\begin{aligned}
& u_{k I}(x, w)=\sum_{r_{1}, \ldots-1}^{m-1} \int_{k}^{1} \ldots \int_{0}^{1} z_{r_{1}}\left(y_{1}\right) \ldots z_{r_{k}}\left(y_{k}\right) \frac{l}{H_{r_{1}} \ldots r_{k}}\left(x ; y_{1}, \ldots, y_{k}\right) d y_{1} \ldots d y_{k} \\
& \lambda_{k I}(\omega)=\sum_{r_{1}, \ldots, r_{k}=0}^{m-1} \int_{0}^{1} \int_{0} F_{r_{1}}\left(y_{1}\right) \ldots F_{r_{k}}\left(y_{k}\right) I_{r_{1} \ldots r_{k}}\left(y_{1}, \ldots, y_{k}\right) d y_{1} \ldots d y_{k} \\
& (k \geq 1) \text { where }
\end{aligned}
$$

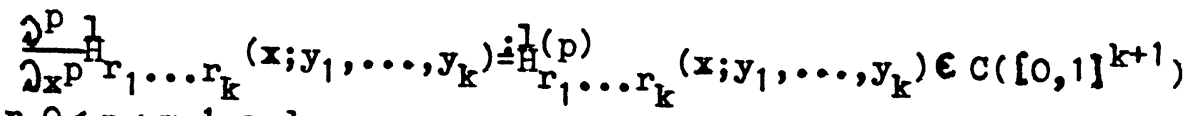

$$
\begin{aligned}
& \text { for } 0 \leqslant \mathrm{p} \& \mathrm{~m}-1 \text { and } \\
& F_{r_{1} \ldots r_{k}}\left(y_{1}, \ldots, y_{k}\right) \in c\left([0,1]^{k}\right) \text {. } \\
& \text { Particular we obtain } \\
& F_{r}(y)=\left(w_{I}^{(r)}(y)\right)^{2}, \quad \quad_{r}(x ; y)=-\frac{\partial^{r} G_{I}(x, y)}{\partial y^{r}} w_{I}^{(r)}(y)
\end{aligned}
$$


if $G_{I}(x, y)$ is the generalized Green function to the operator $\langle I\rangle-h_{I}$ and the boundary conditions $u^{(k)}(0)=u^{(k)}(1)=0$ for $k=0,1, \ldots, m-1$.

For the following we define the notation

$$
\Lambda_{I i}(w): \begin{cases}\lambda_{I}(w) & \text { for } i=0 \\ n_{I}\left(x_{i}, w\right) & \text { for } i \neq 0\end{cases}
$$

if $x_{i} \in[0,1]$ for $i=1,2, \ldots$ are given numbers. Then we obtain, as giren above,

$$
\Lambda_{I i}(\omega)=\sum_{k=0}^{\infty} \Lambda_{k I i}(\omega) \quad \text { (convergence } a_{. s_{0}} \text { ) }
$$

when we additionalIy assume the convergence of $\sum_{k=0}^{\infty} u_{k I}(x, w)$ a.s. for each $x \in[0,1]$. Hence we have

$\Lambda_{k I i}(w)=\sum_{r_{1}, \ldots, r_{k}=0} \int_{0}^{1} \ldots \int_{\delta}^{1} F_{r_{1}}\left(y_{1}\right) \ldots \bar{f}_{r_{k}}\left(y_{k}\right) z_{r_{1} \ldots r_{k}, i}\left(y_{1}, \ldots, y_{k}\right) d y_{1} \ldots d y_{k}$ with

$$
\stackrel{I}{G}_{r_{1} \ldots r_{k}, i}\left(y_{1}, \ldots, y_{k}\right)= \begin{cases}\frac{1}{F_{r_{1}}} \ldots \ldots r_{k}\left(y_{1}, \ldots, y_{k}\right) & \text { for } i=0 \\ \frac{1}{r_{1} \ldots r_{k}}\left(x_{i} ; y_{1}, \ldots, y_{k}\right) & \text { for } i \neq 0 .\end{cases}
$$

With these notations we prove the following theorem 7 .

THEOREM 7. Be $x_{i} \in[0,1], h_{i j}(w)=\sum_{k=0} \Lambda_{k I i}(\omega)$ and Iet $\bar{f}_{r e}(x, w)$ be aequences of Independent, weakly correlated processes with the correlation length $\& \downarrow t$ where

$$
\left\langle z_{r e}(x) I_{r e}(y)\right\rangle=\left\{\begin{array}{cc}
R_{r e}(x, y) & \text { for } y \in K_{\varepsilon}(x) \\
0 & \text { for } y \notin K_{c}(x)
\end{array}\right.
$$

and $\operatorname{Iim}_{t \rightarrow 0} \frac{1}{\varepsilon} \int_{-l}^{t} R_{r e}(x, x+y) d y=a_{r}(x)$ uniformly in $x$. Then the random rector

$$
\frac{1}{r}\left(\Lambda_{I_{1} i_{1}}-\Lambda_{0 I_{1} i_{1}}, \ldots, \Lambda_{I_{8} i_{8}}-\Lambda_{o I_{8} i_{8}}\right)^{T}
$$

converges in the distribution to a Gaussian random vector

$$
\xi_{I_{1} i_{1}}, \xi_{I_{2} I_{2}} \cdots \xi_{I_{s} i^{2}} I^{T} \text { with } \xi_{I_{p_{p}}}=\sum_{t=0}^{m-1} \xi_{t I_{p} I_{p}} \text {. }
$$

The random vector $I_{t} \dot{*}\left(S_{t I_{1} i_{1}}, \ldots, S_{t I_{s} i_{s}}\right)^{T}, t=0,1, \ldots, m-1$, are Independent and we have

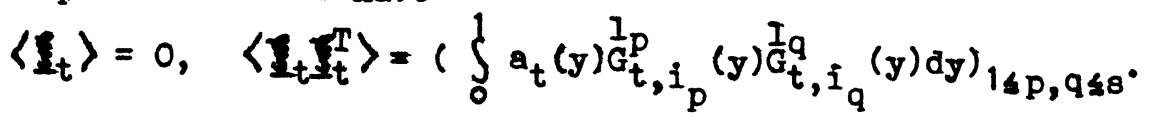


PROOF. We calculate the $k$-th moment

$$
\frac{1}{\sqrt{\varepsilon}}\left\langle\left(\Lambda_{I_{a_{1}}{ }^{i} a_{1}}^{(n)}-\Lambda_{O I_{a_{1}}{ }^{i} a_{1}}\right) \ldots\left(\Lambda_{I_{a_{k}}{ }^{i} a_{k}}-\Lambda_{\sigma I_{a_{k}}{ }^{i} a_{k}}\right)\right\rangle
$$

with $a_{q} \in\{1, \ldots, s\}, \Lambda_{I i}^{(n)}(w)=\sum_{k=0}^{n} \Lambda_{k l i}(w)$ and show that this moment converges to the adequate $k$-th moment of $\left\{\xi_{I_{1} I_{1}}, \ldots, \xi_{I_{s} i_{s}}\right\}$.

At first we calculate the order of a term of the form $P_{p \varepsilon}=\sum_{r_{1}, \ldots, r_{p}}^{m-1} \int_{00}^{1} \ldots \int_{0}^{1} \bar{f}_{r_{1} \varepsilon}\left(y_{1}\right) \ldots \bar{f}_{r_{p} \varepsilon}\left(y_{p}\right) I_{r_{1}} \ldots r_{p}\left(y_{1}, \ldots, y_{p}\right) d y_{1} \ldots d y_{p}$ as $\&$ if $\mathrm{L}_{\mathrm{r}_{1} \ldots \mathrm{r}_{\mathrm{p}}}$ satisfies the condition $\left|\mathrm{L}_{\mathrm{r}_{1} \ldots \mathrm{r}_{\mathrm{p}}}\left(\mathrm{y}_{1}, \ldots, \mathrm{y}_{\mathrm{p}}\right)\right| \leqslant \mathrm{C}$. Because of the independence of the processes $x_{i \varepsilon}(x, w)$, we must deal with terms of the form

$$
\begin{aligned}
& Q_{\varepsilon}=\int_{0}^{1} \ldots \int_{0}^{1}\left\langle\bar{f}_{o \varepsilon}\left(g_{1}\right) \ldots \bar{f}_{o \varepsilon}\left(g_{p_{0}}\right)\right\rangle\left\langle\bar{f}_{1 \varepsilon}\left(y_{1}\right) \ldots \bar{f}_{1 \varepsilon}\left(y_{p_{1}}\right)\right\rangle \cdot \ldots \cdot
\end{aligned}
$$

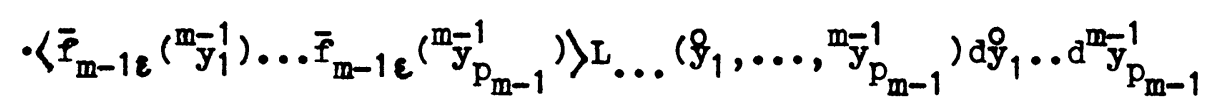

when we want to calculate the order of $\mathrm{P}_{\mathrm{p} \varepsilon}$. It follows

$$
\begin{aligned}
& \left|Q_{\varepsilon}\right| \leqslant c \int_{0}^{1} \ldots \int_{0}^{1} \mid\left\langle\bar{f}_{o \varepsilon}\left(\dot{y}_{1}\right) \ldots \bar{f}_{o \varepsilon}\left(\left(_{p_{p}}\right)\right\rangle\right| d \dot{y}_{1} \ldots d \dot{y}_{p_{0}} \cdot \ldots \cdot \\
& \text { - } \left.\int_{0}^{1} \ldots \int_{0}^{1} K \bar{f}_{m-1 \varepsilon}\left({ }^{m-1} \bar{y}_{1}\right) \ldots \bar{f}_{m-1 \varepsilon}\left({ }^{m-1} \bar{y}_{p_{m-1}}\right)\right\rangle \mid d^{m} \bar{y}_{1}^{1} \ldots d^{m-1} y_{p_{m-1}}^{1}
\end{aligned}
$$

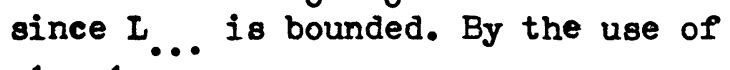

$$
\int_{0}^{1} \ldots \int_{0}^{1}\left|\left\langle\bar{f}_{q \varepsilon}\left(g_{1}\right) \ldots \bar{f}_{q \varepsilon}\left(g_{p_{q}}\right)\right\rangle\right| d g_{1} \ldots d y_{p_{q}}= \begin{cases}o\left(\varepsilon p_{q} / 2\right) & \text { for } p_{q} \text { even } \\ o\left(\varepsilon\left(p_{q}+1\right) / 2\right) & \text { for } p_{q} \text { odd }\end{cases}
$$

(see the proof of the adequate theorem in [6] to the theorem 3 of this paper) we obtain an estimation of the order of $Q_{\varepsilon}$ and hence we obtain the estimate of $\mathrm{P}_{\mathrm{pe}}$

$$
\left|P_{p \varepsilon}\right| \leqslant \begin{cases}o(\varepsilon p / 2) & \text { for } p \text { even } \\ O\left(\varepsilon^{(p+1) / 2}\right) & \text { for } p \text { odd. }\end{cases}
$$

With this result we can now show considering theorem 6

$$
\begin{aligned}
& \frac{1}{\sqrt{\varepsilon}^{K}}\left\langle\left(\Lambda_{I_{a_{1}}{ }^{i} a_{1}}^{(n)}-\Lambda_{o I_{a_{1}}{ }^{i} a_{1}}\right) \ldots\left(\Lambda_{I_{a_{k}}{ }^{(n)} a_{k}}-\Lambda_{o I_{a_{k}}{ }^{i} a_{k}}\right)\right\rangle \\
& =\frac{1}{\sqrt{\varepsilon}^{k}}\left\langle\left(\sum_{p=1}^{n} \Lambda_{p_{1} a_{1}{ }^{i} a_{1}}\right) \ldots\left(\sum_{p=1}^{n} \Lambda_{p l_{a_{k}}{ } a_{k}}\right)\right\rangle
\end{aligned}
$$


and hence

$$
=\left\{\begin{array}{cc}
\frac{1}{\sqrt{\varepsilon}}\left\langle\Lambda_{11_{a_{1}}{ }^{i} a_{1}}\right. \\
0(\sqrt{\varepsilon}) & \text { for } k \text { odd }
\end{array}\right.
$$

$$
\begin{aligned}
& \text { Iim } \frac{1}{\sqrt{\varepsilon}}\left\langle\left(\Lambda_{I_{a_{1}}{ }^{i} a_{1}}^{(n)}-\Lambda_{o I_{a_{1}} i_{a_{1}}}\right) \ldots\left(\Lambda_{I_{a_{k}}{ }^{i} a_{k}}^{(n)}-\Lambda_{o I_{a_{k}}{ }^{i} a_{k}}\right)\right\rangle \\
& =\left\{\begin{array}{cc}
\operatorname{Iim} \frac{1}{\sqrt{\varepsilon} k}\left\langle\bar{\lambda}_{a_{1}} \bar{\lambda}_{a_{2}} \cdots \pi_{a_{k}}\right\rangle & \text { for } k \text { even } \\
0 & \text { for } k \text { odd }
\end{array}\right.
\end{aligned}
$$

with $\pi_{a_{q}} \doteq \Lambda_{1 I_{a_{q}} i_{a_{q}}}$. By $\Lambda_{11 i}=\sum_{t=0}^{m-1} \int_{0}^{1} z_{t}(y)^{\frac{I}{G}}{ }_{t, i}(y)$ dy and by similar considerations as in the proof of theorem 5 we obtain the theorem for $\Lambda_{i i}^{(n)}$.

We will show in the following considerations that the theorem 7 is also right for $\frac{1}{k}\left(\Lambda_{I_{1} i_{1}}-\Lambda_{o I_{1} i_{1}}, \ldots, \Lambda_{I_{s} i_{s}}-\Lambda_{o I_{s} i_{s}}\right)^{T}$ instead of $\frac{1}{F^{2}}\left(\Lambda_{I_{1} i_{1}}^{(n)}-\Lambda_{o l_{1} i_{1}}, \ldots, \Lambda_{1_{s} i_{s}}^{(n)}-\Lambda_{o l_{s} i_{s}}\right)^{T}$. For this we denote the convergence

$$
\lim _{n \rightarrow \infty} \frac{1}{\varepsilon}\left\langle\left(\Lambda_{I i}^{(n)}-\Lambda_{I i}\right)^{2}\right\rangle=0
$$

uniformly in $\varepsilon$. We will deal with the idea of this proof at the boundary value problems because we use for the proof fundamental estimations from the perturbation theory.

Then from this convergence in $\mathrm{I}_{2}$-mean it follows the convergence in probability uniformly in

and then

$$
\operatorname{P-Iim}_{n \rightarrow \infty} \frac{1}{\varepsilon}\left(\Lambda_{1 i}^{(n)}-\Lambda_{o I i}\right)=\frac{1}{\sqrt{\varepsilon}}\left(\Lambda_{1 i}-\Lambda_{o l i}\right)
$$

$$
\lim _{n \rightarrow \infty} P\left(\frac{1}{\sqrt{\varepsilon}}\left(\Lambda_{I i}^{(n)}-\Lambda_{o l i}\right)<t\right)=P\left(\frac{1}{\sqrt{\varepsilon}}\left(\Lambda_{1 i}-\Lambda_{o l i}\right)<t\right)
$$

uniformly in $\varepsilon$. We have shown in a first part of this proof

$$
\lim _{\text {to }} F_{\varepsilon s}^{(n)}\left(t_{1}, \ldots, t_{s}\right)=\phi_{s}\left(t_{1}, \ldots, t_{s}\right) \quad \text { for all } n \geq 1
$$

if we set

$$
\begin{aligned}
& F_{\varepsilon s}^{(n)}\left(t_{1}, \ldots, t_{s}\right)=P\left(\frac{1}{\sqrt{\varepsilon}}\left(\Lambda_{I_{1} i_{1}}^{(n)}-\Lambda_{o I_{1} i_{1}}\right)<t_{1}, \ldots, \frac{1}{\sqrt{\varepsilon}}\left(\Lambda_{I_{s} i_{s}}^{(n)}-\Lambda_{b I_{s} i_{s}}\right)<t_{s}\right) \\
& \phi_{s}\left(t_{1}, \ldots, t_{s}\right)=P\left(\xi_{I_{1} i_{1}}<t_{1}, \ldots, \xi_{I_{s} i_{s}}<t_{s}\right) .
\end{aligned}
$$


Let $F_{\varepsilon s}\left(t_{1}, \ldots, t_{s}\right)$ be the distribution function of $\frac{1}{r} a_{I_{1} i_{1}}-\Lambda_{o I_{1} i_{1}}$, $\left.\cdots, \Lambda_{I_{s} s}-\Lambda_{o I_{s}}\right){ }^{T}$. Hence we obtain from relations as in Eq. (8) $\left|F_{\varepsilon s}^{(n)}\left(t_{1}, \ldots, t_{s}\right)-F_{\varepsilon s}\left(t_{1}, \ldots, t_{s}\right)\right|<\eta$

for all $n \geq n_{0}(\eta)$ uniformly in $\varepsilon$ and with Eq. (9)

$$
\phi_{s}\left(t_{1}, \ldots, t_{s}\right)-\eta \leqslant \overline{\frac{I i m}{\varepsilon+0}} F_{\varepsilon s}\left(t_{1}, \ldots, t_{s}\right) \leqslant \phi_{s}\left(t_{1}, \ldots, t_{s}\right)+\eta
$$

for every $\eta>0$. Then it follows

$$
\lim _{\text {eto }} F_{\varepsilon s}\left(t_{1}, \ldots, t_{s}\right)=\phi_{s}\left(t_{1}, \ldots, t_{s}\right)
$$

and by it the theorem 7 .

Now we consider a few important special cases of theorem 7:

(a) $\frac{1}{\sqrt{c}}\left(\lambda_{1}(w)-\mu_{1}\right) \frac{\text { in the distribution }}{\text { to }} \xi_{10}$, where $\xi_{10}$ is a Gaussian random variable with the parameters

$$
\left\langle\xi_{10}\right\rangle=0,\left\langle\xi_{10}^{2}\right\rangle=\sum_{t=0}^{m-1} \int_{0}^{1} a_{t}(y)\left(w_{1}^{(t)}(y)\right)^{4} d y .
$$

(b) $\frac{1}{\sqrt{\varepsilon}}\left(u_{1}(x, w)-w_{I}(x)\right) \frac{\text { in the distribution }}{2+0} \eta_{I}(x)$, where $\eta_{I}(x)$ is a Gaussian process with the parameters

$$
\begin{aligned}
& \left\langle\eta_{I}(x)\right\rangle=0, \\
& \left\langle\eta_{I}(x) \eta_{I}(y)\right\rangle=\sum_{t=0}^{m-1} \int_{0}^{1} a_{t}(z) \frac{\partial^{t} G_{I}(x, z)}{\partial_{z}^{t}} \frac{\partial^{t} G_{I}(y, z)}{\partial z}\left(w_{I}(t)(z)\right)^{2} d z .
\end{aligned}
$$

By theorem 7 the distribution function of the random vector $\underline{U}=\frac{1}{\sqrt{\varepsilon}}\left(u_{1}\left(x_{1}\right)-w_{1}\left(x_{1}\right), \ldots, u_{1}\left(x_{k}\right)-w_{1}\left(x_{k}\right)\right)^{T}$ converges as $c$ to to the distribution function $\phi_{x_{1} \ldots x_{k}}\left(t_{1}, \ldots, t_{k}\right)$ of the random vector $\left(\xi_{11}, \ldots, \xi_{I k}\right)^{\mathrm{T}}$ which is a Gaussian random vector with

$$
\begin{aligned}
\left\langle J_{I p} s_{I q}\right\rangle & =\sum_{t=0}^{m-1}\left\langle E_{t p} J_{t I q}\right\rangle=\sum_{t=0}^{m-1} \int_{0}^{1} a_{t}(y) G_{t, p}(y) G_{t, q}(y) d y \\
& =\sum_{t=0}^{m-1} \int_{0}^{1} a_{t}(y) \frac{\partial^{t}}{\partial t^{t} G_{I}}\left(x_{p}, y\right) \frac{\partial^{t}}{\partial t^{t} G_{I}}\left(x_{q}, y\right)\left(w_{I}(t)(y)\right)^{2} d y .
\end{aligned}
$$

Hence the case (b) is proved.

$$
\begin{aligned}
& \text { (c) } \frac{1}{\sqrt{\varepsilon}}\left(\lambda_{I_{1}}-\mu_{I_{1}}, \ldots, \lambda_{I_{r}}-\mu_{I_{r}}, u_{I_{r+1}}(x)-w_{I_{r+1}}(x), \ldots, u_{I_{k}}(x)-w_{I_{k}}(x)\right)^{T} \\
& \underset{\text { in the distribution }}{\stackrel{\text { in }}{\longrightarrow}}\left(\xi_{I_{1},}, \ldots, \xi_{I_{r_{T}},}, \eta_{I_{I+1}}(x), \ldots, \eta_{I_{k}}(x)\right)^{T} \text {, } \\
& \text { where } \boldsymbol{S}(x)=\left(\xi_{I_{1},}, \ldots, \xi_{I_{r},}, \eta_{I_{r+1}}(x), \ldots, \eta_{I_{k}}(x)\right)^{T} \text { is a Gaussian }
\end{aligned}
$$


process with $\langle\underline{\underline{S}}(x)\rangle=0$ and

$\left\langle\underline{\mathfrak{S}}(\mathrm{x}) \underline{\mathrm{S}}(\mathrm{y})^{\mathrm{T}}\right\rangle=$

$=\sum_{t=0}^{m-1} \int_{0}^{1} a_{t}(z)\left(\begin{array}{ll}\left.\left(w_{1}^{(t)}(z) w_{1}^{(t)}(z)\right)^{2}\right)_{1 \leqslant p, q \leqslant r}\left(v_{q p}^{t}(y, z)\right)_{1 \leqslant p \leqslant r} \\ \left(v_{p q}^{t}(x, z)\right)_{\substack{r+1 \leqslant p \leqslant k \\ 1 \leqslant q \leqslant r}} & \left(w_{p q}^{t}(x, y, z)\right)_{r+1 \leqslant p, q \leqslant k}\end{array}\right) d z$

with $v_{p q}^{t}(x, z)=-\frac{\partial^{t} G_{I_{p}}(x, z) r}{\partial z}\left(w_{I_{q}}^{(t)}(z)\right)^{2} w_{I_{p}}^{(t)}(z)$

$w_{p q}^{t}(x, y, z)=\frac{\partial^{t} G_{I_{p}}(x, z)}{\partial z^{t}} \frac{\partial^{t} G_{I_{q}}(y, z)}{\partial z^{t}} w_{I_{p}}^{(t)}(z) w_{I_{q}}{ }^{t}(z)$.

This case (c) also follows from theorem 7.

3.2. The results of this section can also be used of eigenvalue problems of the form

$$
\mathrm{Lu}=\lambda \mathrm{h}(\mathbf{x}, \boldsymbol{w}) \mathrm{u}, \quad U_{i}[\mathrm{u}]=0, i=1,2, \ldots, 2 \mathrm{~m}, 0 \leqslant \mathbf{x} \leqslant 1 .
$$

The operator $I$ is a deterministic differential operator of the order $2 m$

$$
L u \stackrel{m}{=} \sum_{r=0}^{m}(-1)^{r}\left[f_{r}(x) u^{(r)}\right]^{(r)}
$$

where the coefficient $f_{r}(x)$ is continuous differentiable of $r$-th order. It is for the stochastic process $h(x, w)$ the equation $h(x, w)=$ $h_{0}(x)+g(x, w)$. Let $h_{0}(x)$ be positive and let the process $g(x, w)$ be a weakly correlated process of the correlation length $\varepsilon$ with $|g(x, \omega)| \leqslant \eta \quad$ ( $\eta$ sufficient smalI). The deterministic boundary conditions $U_{i}[u]=0, i=1,2, \ldots, 2 m$, are constituted in that manner that the problem (11) is selfadjoint and positive definite.

Ey the made suppositions the averaged problem to (11) Lw $=\mu_{0} \mathrm{w}$, $U_{i}[w]=0, i=1,2, \ldots, 2 m$, possesses enumerable many positive eigenvalues $0<\mu_{1} \leqslant \mu_{2} \leqslant \ldots$. Let all eigenvalues $\mu_{I}$ be simple. The eigenfunctions $w_{1}(x)$ are assumed to be orthonormal in $I_{2}(0,1)_{h_{0}}$, i.e. we have for $k, 1=1,2, \ldots$

$$
\left(w_{k}, w_{I}\right)_{h_{0}} \dot{=} \int_{0}^{l} w_{k}(x) w_{1}(x) h_{0}(x) d x=\delta_{k I} .
$$

We define as in section 3.1. 


$$
\Lambda_{1 i}(\omega)= \begin{cases}\lambda_{I}(\omega) & \text { for } i=0 \\ u_{I}\left(x_{i}, \omega\right) & \text { for } i \neq 0\end{cases}
$$

with $x_{i} \in[0,1]$. Then a development exists of $\Lambda_{I i}(\omega)$ (see /5/) $\Lambda_{1 i}(\omega)=$ $\sum_{k=0}^{\infty} \Lambda_{k I_{i}}(\omega)$ for which we assume the convergence of $\sum_{k=0}^{\infty}\left\langle\left|\Lambda_{k I i}\right|\right\rangle$. It is $\Lambda_{\text {oli }}=\left\{\begin{array}{ll}\mu_{I} & \text { for } i=0 \\ w_{I}\left(x_{i}\right) & \text { for } i \neq 0\end{array}\right.$ and $\Lambda_{1 I i}= \begin{cases}-\mu_{1} b_{I I} & \text { for } i=0 \\ \mu_{1} \int_{0} G_{I}\left(x_{i}, y\right) g(y, w) w_{I}(y) d y & \text { for } i \neq 0\end{cases}$ where $G_{1}(x, y)$ denotes the generalized Green function to $I-\mu_{1} h_{0}$ and the boundary conditions $U_{i}, i=1,2, \ldots, 2 m$, and $b_{I I}(w) \stackrel{1}{=} \int_{0}^{1}\left(w_{I}(y)\right)^{2} g(y, w) d y$. By

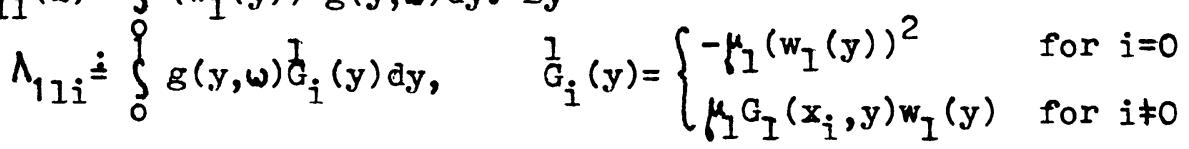

we can formulate a to theorem 7 adequate theorem.

THEOREM 8. Let $x_{1}, x_{2}, \ldots$ be from $[0,1]$ and let $g_{\varepsilon}(x, w)$ be a sequence of weakly correlated processes with the correlation length $\varepsilon$ and

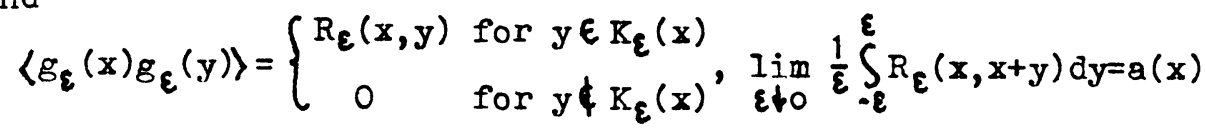

$(a(x) \neq 0)$ uniformly in $x$. Then the random vector

$$
\frac{1}{\sqrt{\varepsilon}}\left(\Lambda_{I_{1} i_{1}}-\Lambda_{\text {ol } i_{1}}, \ldots, \Lambda_{I_{s} i_{s}}-\Lambda_{\text {ol }} i_{s}\right)^{T}
$$

formed from the stochastic eigenvalue problem (11) with $g_{\ell}(x, w)$ converges in the distribution to a Gaussian random vector

$$
\begin{aligned}
\left(\xi_{I_{1} i_{1}}, \cdots, \xi_{I_{s} i_{s}}\right)^{T} \text { with } & =0,\left\langle\xi_{I_{p}{ }_{p}} \xi_{I_{q} i_{q}}\right\rangle=\int_{0}^{1} a(y) G_{i_{p}}^{I_{p}}(y) G_{i_{q}}^{I_{q}}(y) d y .
\end{aligned}
$$

REMARK. We can also prove such a theorem for the more general stochastic eigenvalue problem

$$
L u+I_{1}(w) u=\lambda\left(h_{0} u+M_{1}(w) u\right), U_{i}[u]=0, i=1,2, \ldots, 2 m, 0 \leqslant x \leqslant 1 .
$$

Here the operator $\mathrm{I}$ is a deterministic differential operator like in Eq. (12) and

$$
I_{1}(w) u=\sum_{k=0}^{m-1} a_{k}(x, w) u^{(k)}, M_{1}(w) u=\sum_{k=0}^{m-1} b_{k}(x, w) \dot{u}^{(k)}
$$


with $\left\langle I_{1}(w)\right\rangle=\left\langle M_{1}(w)\right\rangle=0$ where the coefficients $a_{k}(x, w), b_{k}(x, w)$ are independent, weakly correlated processes. Perturbation serieses reIativ to $L_{1}(\omega), M_{1}(w)$ form the basic. These serieses were deduced in [5].

3.3. We regard the stochastic eigenvalue problem

$$
-u^{n}+a(x, w) u=\lambda(1+b(x, w)) u, u(0)=u(1)=0
$$

where $a(x, w), b(x, w)$ denote independent, weakly correlated processes of the correlation length $\varepsilon$. The averaged problem to (13) is

$$
-w^{n}=\mu w, w(0)=w(1)=0 .
$$

This averaged problem possesses the simple eigenvalues $h_{1}=(1 \pi)^{2}$ and the eigenfunctions $w_{1}(x)=\sqrt{2} \sin (1 \pi x)$. It is for the processes $a(x, \omega)$ and $b(x, \omega)$

$$
\begin{gathered}
\langle a(x) a(y)\rangle=R_{a \varepsilon}(x, y), \quad\langle b(x) b(y)\rangle=R_{b \varepsilon}(x, y) \\
\left(R_{a \varepsilon}(x, y)=R_{b \varepsilon}(x, y)=0 \text { for } y \notin K_{\varepsilon}(x)\right) \text { and } \\
\quad \operatorname{Iim} \frac{1}{\varepsilon} \int_{-\varepsilon}^{R_{a \varepsilon}} R_{a, x}(x, y) d y=\alpha(x), \operatorname{Iim}_{\varepsilon} \frac{1}{\varepsilon} \int_{-\varepsilon}^{\varepsilon} R_{b \varepsilon}(x, x+y) d y=\beta(x) .
\end{gathered}
$$

By the supposition that $|a(x, w)|$ and $|b(x, w)|$ are smalI we obtain the development (with the notation as above given)

$$
\begin{gathered}
\Lambda_{l i}(w)=\sum_{k=0}^{\infty} \Lambda_{k l i}(\omega) \quad \text { where } \\
\Lambda_{\text {oli }}=\left\{\begin{array}{ll}
\mu_{l} & \text { for } i=0 \\
w_{I}\left(x_{i}\right) & \text { for } i \neq 0
\end{array} \text { is and with } G_{i}(y)= \begin{cases}\left(w_{I}(y)\right)^{2} & \text { for } i=0 \\
-G_{I}\left(x_{i}, y\right) w_{I}(y) & \text { for } i \neq 0\end{cases} \right.
\end{gathered}
$$

the formula $\Lambda_{11 i}=\int_{0}^{1}\left(a(y)-\mu_{1} b(y)\right) G_{i}^{l}(y) d y$ is right. It is

$$
G_{I}(x, y)=\left\{\begin{array}{c}
\frac{1}{I \pi}\left\{\left[\frac{1}{2 I \pi} \sin (1 \pi x)-x \cos (1 \pi x)\right\} \sin (1 \pi y)+(1-y) \cos (I \pi y) \sin (1 \pi x)\right] \\
\text { for } 0 \leqslant x<y \leqslant 1 \\
\frac{1}{I \pi}\left\{\left[\frac{1}{2 I \pi} \sin (1 \pi x)+(1-x) \cos (1 \pi x)\right\} \sin (1 \pi y)-y \cos (1 \pi y) \sin (I \pi x)\right] \\
\text { for } 0 \leqslant y<x \leqslant 1 .
\end{array}\right.
$$

By theorem 7 the random vector $\frac{1}{\sqrt{\varepsilon}}\left(\Lambda_{I_{1} i_{1}}-\Lambda_{O I_{1} i_{1}}, \ldots, \Lambda_{I_{s} i_{s}}-\Lambda_{o l_{s} i_{s}}\right)^{T}$ converges in the distribution to the Gaussian random vector $\left(\xi_{I_{1} i_{1}}, \ldots, \xi_{I_{s} i_{s}}\right)^{T}$ with 
$\left.\left.\left\langle\xi_{I_{p} p_{p}}\right\rangle=0,\left\langle\xi_{I_{p} p_{p}} \xi_{I_{q} q_{q}}\right\rangle=\int_{0}^{1}\left(\alpha(y)+\mu_{I_{p}} \mu_{I_{q}} \beta(y)\right)\right)_{I_{p}}^{I_{p}}(y)\right)_{i_{q}}^{I_{q}}(y) d y$.

Farticulary we obtain:

(a) For $F_{\varepsilon}^{I k}(t, s) \dot{=} P\left(\frac{1}{\sqrt{\varepsilon}}\left(\lambda_{I}-\mu_{I}\right)<t, \frac{1}{\sqrt{\varepsilon}}\left(\lambda_{k}-\mu_{k}\right)<s\right)$ and $\phi^{I k}(t, s)=$ $P\left(I_{10}<t, \xi_{k 0}<s\right)$ (Gaussian distribution) we have

$\lim _{F^{I k}(t, s)=\phi^{I k}(t, s) .}$.
with $\left\langle\xi_{p o}\right\rangle=0,\left(\left\langle\xi_{p o} \xi_{q o}\right\rangle\right)_{p, q \in\{I, k\}}=\left(\int_{0}\left(\alpha+\mu_{p} \mu_{q} \beta\right) w_{p}^{2} w_{q}^{2} d y\right)_{p, q \in\{I, k\}}$

Particulary for wide-sense stationary, weakly correlated processes it is

$$
\left(\left\langle\xi_{p o} \xi_{q 0}\right\rangle\right)_{p, q \in\{I, k\}}=\left(\begin{array}{cc}
\frac{3}{2}\left(\alpha+\mu_{p}^{2} \beta\right) & \alpha+\mu_{p} \mu_{q} \beta \\
\alpha+\mu_{p} \mu_{q} \beta & \frac{3}{2}\left(\alpha+\mu_{q}^{2} \beta\right)
\end{array}\right) \text {. }
$$

The variation of the limit distribution depends on the number of the eigenvalues

$$
\left\langle\xi_{I 0}^{2}\right\rangle=\frac{3}{2}\left(\alpha+\mu_{I}^{2} \beta\right)
$$

if $b \neq 0$ and $a(x, \omega), b(x, \omega)$ are wide-sense stationary, weakly correlated processes. This is reflected in a melt of the eigenvalues with increasing number (see Fig.2).

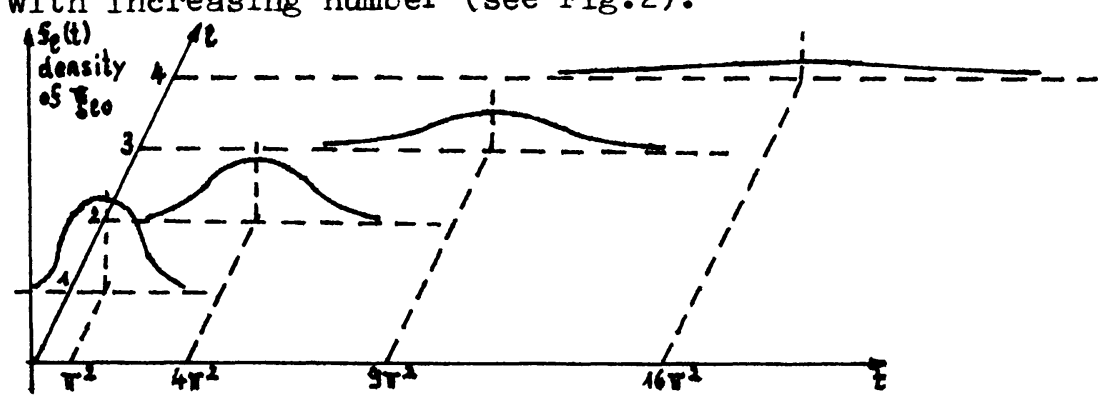

Fig.2

$B y b \equiv 0$ the variation of the limit distribution does not depends on the number of the eigenvalues. In this case the correlation $\left\langle\xi_{10}\right\rangle=\alpha$ for $k \neq I$ is independent of $k$ and 1 . This effect is explicable from the fact that the operator $L(\omega)$ determines the eigenvalues respectively the eigenvalues $\lambda_{I}(\omega)$ for a $\omega_{0}$ determines about the random operator $L(\omega)$ the other eigenvalues $\lambda_{\mathrm{k}}(\omega)$ for this $\omega_{0}$. 
(b) The random vector $\frac{1}{\sqrt{\varepsilon}}\left(u_{1}(x)-w_{1}(x), u_{k}(x)-w_{k}(x)\right)^{T}$ converges in the distribution as $\varepsilon \nmid 0$ to the Gaussian vector process $\left(\eta_{I}(x), \eta_{k}(x)\right)^{T}$ with $\left\langle\eta_{p}(x)\right\rangle=0$, for $p, q \in\{k, I\}$.

$$
\left\langle\eta_{p}(x) \eta_{q}(y)\right\rangle=\int_{0}^{1}\left(\alpha(z)+\mu_{p} \mu_{q} \beta(z)\right) G_{p}(x, z) G_{q}(y, z) w_{p}(z) w_{q}(z) d z
$$

We can also calculate the correlation values of this Gaussian vector process from

$$
\left\langle\eta_{p}(x) \eta_{q}(x)\right\rangle=\operatorname{Iim}_{c \neq 0} \frac{1}{\varepsilon}\left\langle u_{1 p}(x) u_{1 q}(y)\right\rangle
$$

if $u_{1 p}(x, \omega)$ denotes the first term in the development of $a_{p}(x, \omega)$ $w_{p}(x)$ relativ to $a(x, \omega)$ and $b(x, \omega)$. One obtains

$$
\begin{gathered}
u_{1 p}(x, w)=-\frac{1}{p \pi} \int_{0}^{x} \bar{a} w_{p} \sin (p \pi(t-x)) d t+\frac{1}{2 \mu_{p}}\left(x w_{p}^{\prime}(x)-\frac{1}{2} w_{p}(x)\right) \int_{0}^{1} \bar{a} w_{p}^{2} d t \\
-\frac{1}{2 \mu_{p}} w_{p}(x) \int_{0}^{1}(1-t) \bar{a}_{p} w_{p}^{\prime} d t
\end{gathered}
$$

after a few calculations with $\bar{a}(x, \omega)=a(x, \omega)-\mu_{p} b(x, \omega)$ and then from (14) the correlation function of the limit process of $\frac{1}{\sqrt{\varepsilon}}\left(u_{1}-w_{1}\right)$

$$
\begin{aligned}
\left\langle\eta_{I}(x) \eta_{I}(y)\right\rangle=\frac{\bar{\sigma}_{I I}}{8 \mu_{I}^{2}} & \left\{\mu_{I}\left(\frac{x^{2}}{2}+\frac{y^{2}}{2}-y+\frac{1}{3}\right) w_{I}(x) w_{I}(y)+3 x(1-y) w_{I}^{\prime}(x) w_{I}^{\prime}(y)\right. \\
& +\left[w_{I}^{\prime}(x) w_{I}(y)-(1-y) w_{I}(x) w_{I}(y)\right]\left[3-\frac{1}{2}\left(w_{I}^{2}(y)+w_{I}^{2}(x)\right)\right] \\
& \left.+\frac{5}{8} w_{I}(x) w_{I}(y)\left[-5+w_{I}^{2}(y)+w_{I}^{2}(x)\right]\right\} \quad\left(\sigma_{I I}=\alpha+\mu_{I}^{2} \beta\right)
\end{aligned}
$$

and the variation

$\left\langle\eta_{I}^{2}(x)\right\rangle=\frac{{ }^{2}}{4 \mu_{I}^{2}}\left\{3 \mu_{1} x(1-x)+\mu_{I}\left(\frac{1}{6}+2 x(x-1)\right) w_{I}^{2}(x)+\left(x-\frac{1}{2}\right)\left(3-w_{I}^{2}(x)\right) w_{I}^{1}(x) w_{I}(x)\right.$

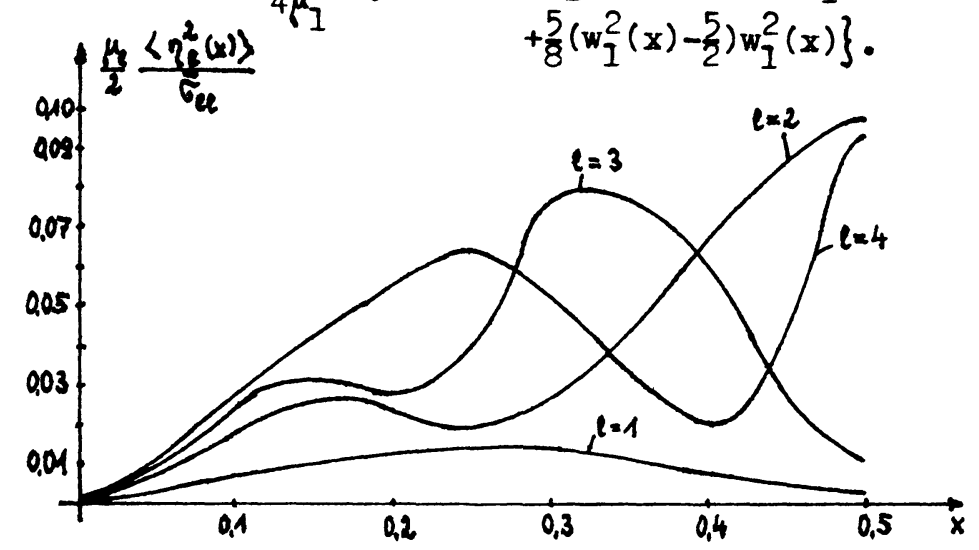

Fig. 3

$\left\langle\eta_{I}^{2}(x)\right\rangle=$

$\left\langle\eta_{I}^{2}(1-x)\right\rangle$ 
The figure 3 shows this variation for the parameters $1=1,2,3,4$. We can make very good statements about the behaviour of the limit process of the efgenfunctions because of the limit process $\eta_{I}(x)$ is a Gausdan process.

4. SIOCHUSTIC BOUNDARY VALUE PROBIEM.

4.1. We consider the stochastic boundary value problem in this rection

$$
\begin{aligned}
& I(\omega) u \neq(-1)^{m}\left[f_{m}(x) u^{(m)}\right]^{(m)}+\sum_{r=0}^{m-1}(-1)^{r}\left[f_{r}(x, w) u^{(r)}\right]^{(r)}=g(x, w) \\
& U_{i}[u]=0, i=1,2, \ldots, 2 m, 0 \leqslant x \leqslant 1 .
\end{aligned}
$$

The boundary conditions are constituted so, that

$$
(\boldsymbol{L}(w) u, \nabla)=\sum_{r=0}^{m} \int_{0}^{1}(-1)^{r}\left[f_{r}(x) u^{(r)}\right]^{(r)} \nabla d x=\sum_{r=0}^{m} \int_{0}^{1} f_{r}(x) u^{(r)}{ }^{(r)} d x
$$

1s: right for all permissible functions $u, v$ (i.e. functions, which possess $2 \mathrm{~m}$ continuous derivatives and fulfil the boundary conditions), i.e. the boundary terms of the integration by parts must be zero. Then the stochastic operator $L(\omega)$ is symetric relativ to all permissible functions.

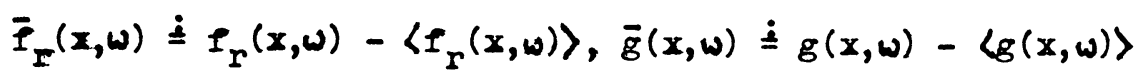

$(0 \leqslant r \leqslant m-1)$ are assumed to be stochastic independent and weakly correlated with the correlation length $c$ and $f_{m}(x) \neq 0$ must be a deterministic continuous function. Further on $\left|F_{r}^{(s)}(x, \omega)\right| \leqslant \eta$ is assumed to be with a small $\eta, s=0,1, \ldots, m-1$, and the processes appearing must be almost everywhere differentiable. The boundary ralue problem (15) can be written in the following form:

$I(w) u=\langle I(w)\rangle u+I_{1}(w) u=\langle g(x, w)\rangle+\bar{g}(x, w), U_{i}[u]=0, i=1,2, \ldots, 2 m$ with $I_{1}(\omega) u=\sum_{r=0}^{m-1}(-1)^{r}\left[\bar{f}_{r}(x, \omega) u(r)\right]^{(r)}$

$$
\left.\langle I(w)\rangle u=(-1)^{m}\left[f_{m}(x) u^{(m)}\right]^{(m)}+\sum_{r=0}^{m-1}(-1)^{r}\left[f_{r}(x, w)\right\rangle u^{(r)}\right]^{(r)} .
$$

We assume that $\langle I(w)\rangle w=0, U_{i}[w]=0$ possess only the trivial solution $w=0$. 
We make for the solution $u(x, w)$ of (15) the statement

$$
u(x, \omega)=\sum_{k=0}^{\infty} u_{k}(x, \omega),
$$

when $u_{k}(x, w)$ denotes the homogeneous part of $k$-th order of $u(x, w)$ in the coefficients $\bar{f}_{r}$ and $\bar{g}$. Substituting this statement in (15) leads to the boundary value problem for $u_{k}(x, w)$

$$
\begin{aligned}
& \langle L\rangle u_{0}=\langle g\rangle ; U_{i}\left[u_{0}\right]=0 \\
& \langle I\rangle u_{1}=\bar{g}-L_{1}(\omega) u_{0} ; U_{i}\left[u_{1}\right]=0 \\
& \langle I\rangle u_{k}=-I_{1}(\omega) u_{k-1} ; U_{i}\left[u_{k}\right]=0 \quad(i=1,2, \ldots, 2 m)
\end{aligned}
$$

Let $G(x, y)$ be the Green function corresponding to $\langle I\rangle$ and to the boundary conditions $U_{i}[]=$.0 then we obtain

$$
u_{0}(x)=\int_{0}^{1} G(x, y)\langle g(y, w)\rangle d y \text {, }
$$

i.e. $u_{0}(x)$ is a deterministic function. With (16) for $u_{1}(x, w)$

$$
\begin{aligned}
u_{1}(x, w) & =\int_{0}^{1} G(x, y)\left\{\bar{g}(y, w)-I_{1}(\omega) u_{0}(y)\right\} d y \\
& =\int_{0}^{1} G(x, y) \bar{g}(y, w) d y-\sum_{r=0}^{m-1} \int_{0}^{1} \bar{f}_{r}(y, w) u_{0}^{(r)}(y) \frac{\partial^{r} G(x, y)}{\partial y^{r}} d y
\end{aligned}
$$

is obtained.

We get the following theorem that is right analog to the theorem 6 for eigenvalue problems:

THEOREM 8. It holds

and for $k=1,2, \ldots$

$$
u_{0}(x)=\int_{0}^{1} G(x, y)\langle g(y, w)\rangle d y
$$

$$
\begin{aligned}
& a_{k}(x, \omega)=\sum_{r_{1}, \ldots, r_{k}=0}^{m-1} \int_{0}^{1} \ldots \int_{0}^{1} f_{r_{1}}\left(y_{1}\right) \ldots z_{r_{k}}\left(y_{k}\right) \frac{1}{r_{r_{1}} \ldots r_{k}}\left(x ; y_{1}, \ldots, y_{k}\right) d y_{1} \ldots d y_{k} \\
& +\sum_{r_{1}, \ldots r_{k-1}}=000 \int_{0}^{1} f_{r_{1}}\left(y_{1}\right) \ldots \bar{f}_{r_{k-1}}\left(y_{k-1}\right) \bar{g}\left(y_{k}\right) \text {. } \\
& \text { - } \stackrel{2}{\mathrm{H}}_{\mathrm{r}_{1} \ldots \mathrm{r}_{\mathrm{k}}}\left(\mathrm{x} ; \mathrm{y}_{1}, \ldots, \mathrm{y}_{\mathrm{k}}\right) \mathrm{d} \mathrm{y}_{1} \ldots \mathrm{dy}_{\mathrm{k}}
\end{aligned}
$$

for the terms $u_{k}(x, w)$ of the development of the solution $u(x, w)$ of the boundary value problem (15) with

$$
\stackrel{1}{H}_{r}(x ; y)=-u_{0}^{(r)}(y) \frac{\partial^{r}}{\partial y^{r}} G(x, y), \quad \stackrel{2}{H}_{r}(x ; y)=G(x, y) \quad \text { and }
$$




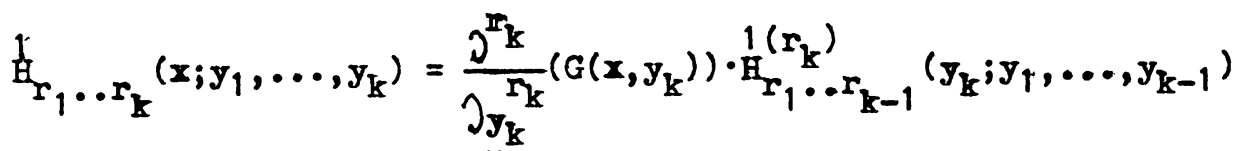

$$
\begin{aligned}
& {\stackrel{2}{\mathrm{H}_{1}}, \ldots r_{\mathrm{k}}}\left(x ; y_{1}, \ldots, \mathrm{y}_{\mathrm{k}}\right)=\frac{\partial^{r_{\mathrm{k}-1}}}{\partial \mathrm{r}_{\mathrm{k}-1}}\left(G\left(x, \mathrm{y}_{\mathrm{k}-1}\right)\right) \text {. } \\
& \cdot{ }_{\mathrm{H}_{1}, \ldots \mathrm{r}_{k-2} \mathrm{r}_{k}}^{2\left(r_{k-1}\right)}\left(y_{k-1} ; y_{\uparrow}, \ldots, y_{k-2}, y_{k}\right) .
\end{aligned}
$$

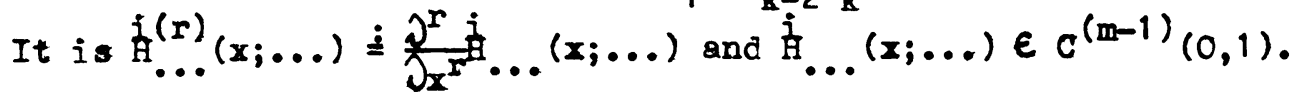
PROOF. The statement follows for $k=1$, from (18). After we have obtained the formula for $\mathrm{k}-1$ then it follows for $\mathrm{k}$ from (17)

$$
\begin{aligned}
& u_{k}(x, w)=-\int_{0}^{1} G(x, y) I_{1}(w) u_{k-1}(y, w) d y \\
& =-\sum_{y=0}^{m-1} \int_{0}^{1} \bar{f}_{r}(y, w) u_{k-1}^{(r)}(y) \frac{\partial_{G}^{r}(x, y)}{\partial_{y}} d y \\
& =-\sum_{r=0}^{m-1} \sum_{r_{1}, \ldots, r_{k-1}=00}^{m-1} \int_{0}^{1} . \iint_{0}^{1} \bar{f}_{r_{1}}\left(y_{1}\right) \ldots \bar{f}_{r_{k-1}}\left(y_{k-1}\right) \frac{\partial r_{G(x, y)}}{\partial y} \text {. } \\
& \text { - } \left.\mathrm{H}_{\mathrm{r}_{1} \ldots \mathrm{r}_{\mathrm{k}-1}}(\mathrm{r}) \mathrm{y}_{1}, \ldots, \mathrm{y}_{\mathrm{k}-1}\right) d \mathrm{y}_{1} \ldots d \mathrm{~d}_{\mathrm{k}-1} \mathrm{dy} \\
& =\sum_{r=0}^{m-1} \sum_{r_{1}, \ldots, r_{k-2}=00}^{m-1} \int_{0}^{1} . \cdot \iint_{0}^{1} \bar{f}_{r_{1}}\left(y_{1}\right) \ldots f_{r_{k-2}}\left(\nabla_{k-2}\right) \bar{x}_{r}(y) \bar{g}\left(y_{k-1}\right) \cdot \\
& \text { - } \frac{\partial^{r} G(x, y)}{\partial y^{r}} L_{r_{1} \ldots r_{k-1}}^{(r)}\left(y ; y_{1}, \ldots, y_{k-1}\right) d y_{1} \ldots d y_{k-1} d y .
\end{aligned}
$$

The theorem 8 is proved.

Now we deal with questions of the convergence of the development. Sinee $\frac{\partial^{r+s} G(x, y)}{\partial x^{r} \partial_{y}^{s}}$ for $x, s=0,1, \ldots, 2 m$ with $0 \leqslant r+s \leqslant 2 m$ is continuous in $0 \leqslant x<y \leqslant 1$ respectively in $0 \leqslant y<x \leqslant 1$, it follows for $(x, y) \in[0,1] x[0,1]$ and the stated $r, s\left|\frac{\partial^{r+s} G(x, y)}{\partial x \partial_{y} s}\right| \leqslant C$, further on we have

$\left|u_{0}^{(r)}(x)\right| \leqslant c_{0} \quad$ for $0 \leqslant x \leqslant 2 m$ and $x \in[0,1]$

$\left|\frac{1}{f_{m}(x)}\right| \leqslant a \quad$ for $x \in[0,1]$ and

$\left|F_{p}^{(r)}(x, w)\right| \leqslant \eta$ almost surely for $0 \leqslant p \leqslant m-1,0 \leqslant r \leqslant p, x \in[0,1]$. $C, C_{0}$ and $a$ are constants. By properties of the Green. function 


$$
\begin{aligned}
& u_{k}^{(2 m)}(x, w)=\int_{0}^{1} \frac{\partial^{2 m_{G}(x, y)}}{\partial x^{2 m}} L_{1} u_{k-1}(y) d y+\frac{(-1)^{m}}{f_{m}(x)} L_{1} u_{k-1}(x) \\
& u_{k}^{(p)}(x, w)=\int_{0}^{1} \frac{\partial^{p_{G}(x, y)}}{\partial x^{p}} L_{1} u_{k-1}(y) d y, \quad p=0,1, \ldots, 2 m-1
\end{aligned}
$$

and therefore

$$
\left|u_{k}^{(p)}(x, w)\right| \leqslant(c+a) \max _{x \in[0,1]}\left|L_{1} u_{k-1}(x)\right| \text { for } p=0,1, \ldots, 2 m \text {. }
$$

Hence we obtain with

$$
\left|I_{1} u_{0}(x)\right| \leqslant \sum_{r=0}^{m-1}\left|\sum_{v=0}^{r}\left(\begin{array}{l}
r \\
v
\end{array}\right) \bar{f}_{r}^{(v)} u_{0}^{(2 r-v)}\right| \in \eta c_{0} \sum_{r=0}^{m-1} \sum_{v=0}^{r}\left(\begin{array}{l}
r \\
v
\end{array}\right)=2^{m} \eta_{0}
$$

for almost all $\omega$ then

$$
\left|u_{1}^{(p)}(x, w)\right| \leqslant 2^{m}(c+a) \eta c_{0} \text { for almost all } w
$$

and through induction for almost all $\omega$

$$
\left|u_{k}(p)(x, w)\right| \leqslant\left(2^{m}(c+a) \eta\right)^{k_{C}}
$$

for $0 \leqslant p \leqslant 2 m$ and $x \in[0,1]$.

Hence $\sum_{k=0}^{\infty}\left|u_{k}(p)(x, w)\right|$ converges almost surely and uniformly in $0 \leqslant x \leqslant 1$ when $|\eta| \leqslant\left(2^{m}(C+a)\right)^{-1}$ for almost all $w$ is right. Then $\sum_{k=0}^{\infty}\left\langle\left|u_{k}^{(p)}(x, w)\right|\right\rangle$ also converges uniformly in $0 \leqslant x \leqslant 1$ with this condition. Let $u_{k}(x, w)$ be calculated by (17) then $u(x, w)=$ $\sum_{k=0}^{\infty} u_{k}(x, \omega)$ is the solution of the stochastic boundary value problem $(15)$. How we formulate the theorem 9.

THEOREM 9. If $\bar{f}_{r}(x, w), \bar{B}(x, w)$ are sequences of independent, weakly correlated processes with the correlation length $e$ t $O$ and

$$
\begin{aligned}
&\left\langle z_{r}(x) \bar{f}_{r}(y)\right\rangle=\left\{\begin{array}{cc}
R_{r \varepsilon}(x, y) & \text { for } y \in K_{\varepsilon}(x) \\
0 & \text { for } y \notin K_{\varepsilon}(x),
\end{array}\right. \\
&\langle\bar{g}(x) \bar{g}(y)\rangle=\left\{\begin{array}{cc}
R_{g \varepsilon}(x, y) & \text { for } y \in K_{\varepsilon}(x) \\
0 & \text { for } y \notin K_{\varepsilon}(x),
\end{array}\right. \\
& \text { Iim } \frac{1}{\varepsilon} \int_{-\varepsilon}^{\varepsilon} R_{r \varepsilon}(x, x+y) d y=a_{r}(y), \text { lim } \frac{1}{\varepsilon} \int_{-\varepsilon}^{\varepsilon} R_{g \varepsilon}(x, x+y) d y=a_{g}(x),
\end{aligned}
$$

then the random vector

$$
\frac{1}{\sqrt{\varepsilon}}\left(u\left(x_{1}, w\right)-u_{a}\left(x_{1}\right), \ldots, u\left(x_{s}, w\right)-u_{0}\left(x_{s}\right)\right)^{T} \quad\left(x_{i} \in[0,1]\right),
$$

which has been constituted by the solution $u(x, w)$ of $(15)$ and the 
solution of the averaged problem to (15), converges in the distribution to a Gaussian random vector

$$
\left(\eta\left(x_{1}, \omega\right), \ldots, \eta\left(x_{s}, \omega\right)\right)^{T}
$$

with $\eta(x, \omega)=\eta_{g}(x, \omega)+\sum_{r=0}^{m-1} \eta_{r}(x, \omega)$. Further on the random vectors

$$
\tilde{\eta}_{g}=\left(\eta_{g}\left(x_{1}, \omega\right), \ldots, \eta_{g}\left(x_{s}, \omega\right)\right)^{T}, \quad \eta_{r}=\left(\eta_{r}\left(x_{1}, \omega\right), \ldots, \eta_{r}\left(x_{g}, \omega\right)\right)^{T} \text {, }
$$

$r=0,1, \ldots, m-1$, are independent Gaussian random vectors with

$$
\begin{aligned}
& \left\langle\tilde{\eta}_{g}\right\rangle=0,\left\langle\tilde{\eta}_{g} \tilde{\eta}_{g}^{T}\right\rangle=\left(\int_{0}^{1} a_{g}(z) G\left(x_{i}, z\right) G\left(x_{j}, z\right) d z\right)_{1 \leqslant i, j \leqslant s} \\
& \left\langle\tilde{\eta}_{r}\right\rangle=0,\left\langle\tilde{\eta}_{r} \tilde{\eta}_{r}^{T}\right\rangle=\left(\int_{0}^{1} a_{r}(z) \frac{\partial^{r} G\left(x_{i}, z\right)}{\partial z^{r}} \frac{\partial^{r} G\left(x_{j}, z\right)}{\partial_{z}^{r}}\left(u_{0}^{(r)}(z)\right)^{2} d z\right)_{1 \leqslant i, j \leqslant s^{*}}
\end{aligned}
$$

REMARK 1. This theorem 9 proves the convergence in the distribution of the processes $\frac{1}{\sqrt{\varepsilon}}\left(u(x, w)-u_{\sigma}(x)\right)$ which has been constituted by the solution $u(x, w)$ of $(15)$ and the solution of the averaged problem to (15) $u_{0}(x)$ to the Gaussian process $\eta(x, w)$ with

$$
\begin{aligned}
& \langle\eta(x, w)\rangle=0 \text { and } \\
& \langle\eta(x) \eta(y)\rangle=\int_{0}^{1} a_{g}(z) G(x, z) G(y, z) d z \\
& +\sum_{r=0}^{m-1} \int_{0}^{1} a_{r}(z) \frac{\partial^{r} G(x, z)}{\partial z^{r}} \frac{\partial^{r} G(y, z)}{\partial z^{r}}\left(u_{0}^{(r)}(z)\right)^{2} d z \text {. }
\end{aligned}
$$

PROOF. We put $n(x, w)=\sum_{k=0}^{n} u_{k}(x, w)$. Then we see with similar considerations as in the proof of theorem 7 and with theorem 2

$$
\text { Iim } \begin{aligned}
\frac{1}{\sqrt{\varepsilon}^{p}}\left\langle\left(v^{n}\left(x_{a_{1}}\right)-u_{0}\left(x_{a_{1}}\right) \ldots\left(\frac{n}{u}\left(x_{a_{p}}\right)-u_{0}\left(x_{a_{p}}\right)\right)\right\rangle\right. \\
\quad=\left\{\begin{array}{cc}
\lim \frac{1}{r^{p}}\left\langle u_{1}\left(x_{a_{1}}\right) \ldots u_{1}\left(x_{a_{p}}\right)\right\rangle & \text { for } p \text { even } \\
0 & \text { for } p \text { odd }
\end{array}\right.
\end{aligned}
$$

where $a_{q} \in\{1, \ldots, s\}$. If we set $u_{1}(x, w)=c(x, w)+\sum_{r=0}^{m-1} b_{r}(x, w)$ (see (18)) with $c(x, \omega)=\int_{0}^{1} G(x, y) \bar{g}(y, \omega) d y$

$$
b_{r}(x, w)=-\int_{0}^{1} \bar{f}_{r}(y, w) u_{0}^{(r)}(y) \frac{\partial^{r} G(x, y)}{\partial y^{r}} d y
$$

it follows as in the proof of the theorem 7

$$
\operatorname{Iim}_{\varepsilon \rightarrow 0} \frac{1}{\sqrt{\varepsilon}}\left\langle u_{1}\left(x_{a_{1}}\right) \ldots u_{1}\left(x_{a_{p}}\right)\right\rangle= \begin{cases}\lim A_{\varepsilon} & \text { for } p \text { even } \\ 0 & \text { for } p \text { odd }\end{cases}
$$


where

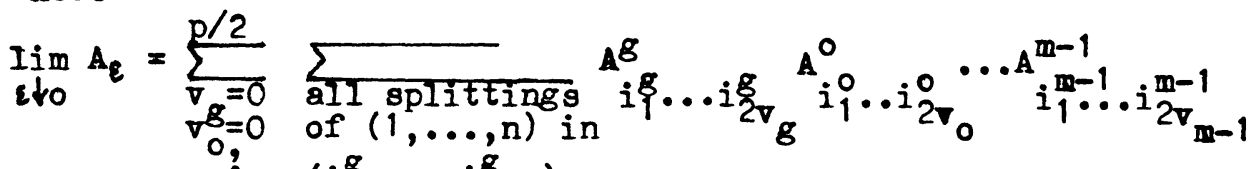

$$
\begin{aligned}
& \text { : } \quad\left(i_{1}^{8}, \ldots, i_{2}^{8}\right)
\end{aligned}
$$

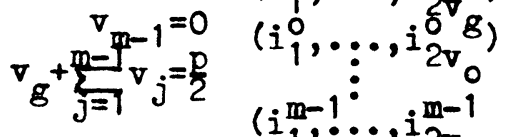

and

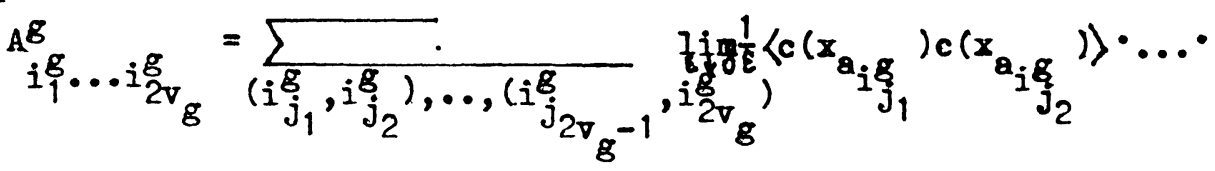

$$
\begin{aligned}
& \text { - } \lim _{\varepsilon \rightarrow} \frac{1}{\varepsilon}\left\langle c\left(x_{a_{i} g}\right) c\left(x_{a_{2 v_{g}}{ }^{-1}}\right)\right\rangle
\end{aligned}
$$

and appropriate for $A^{r}$... with $b_{r}$ instead of $c$. Then the formulas

$$
\begin{aligned}
& \lim _{\text {cimo }} \frac{1}{\varepsilon}\langle c(x) c(y)\rangle=\int_{0}^{1} a_{g}(z) G(x, z) G(y, z) d z \\
& \lim _{\varepsilon \downarrow 0} \frac{1}{\varepsilon}\left\langle b_{r}(x) b_{r}(y)\right\rangle=\int_{0}^{1} a_{r}(z) \frac{\partial^{r} G(x, z)}{\partial_{z}^{r}} \frac{\partial^{r} G(y, z)}{\partial_{z}^{r}}\left(u_{0}(r)(z)\right)^{2} d z
\end{aligned}
$$

imply the statement of the theorem for $\frac{1}{\sqrt{e}}\left(A\left(x_{1}\right)-u_{0}\left(x_{1}\right), \ldots\right.$, $\left.\mathrm{u}\left(\mathrm{x}_{\mathrm{s}}\right)-\mathrm{u}_{0}\left(\mathrm{x}_{\mathrm{s}}\right)\right)^{\mathrm{T}}$.

The complete proof of this theorem follows as in theorem 7 from the uniform convergence relativ to $\varepsilon$

$$
\lim _{n \rightarrow \infty} \frac{1}{\varepsilon}\left\langle(u(x, w)-u(x, w))^{2}\right\rangle=0 \text {. }
$$

We consider $\frac{1}{\sqrt{\varepsilon}}\left\langle u_{p}(x) u_{q}(x)\right\rangle$ with $p, q \geq N_{0}$ to prove formula (20). Then we obtain with theorem 8 and the following estimations

$$
\begin{aligned}
& \left|\frac{1}{\varepsilon}\left\langle u_{p}(x) u_{q}(x)\right\rangle\right| \leq \frac{1}{\varepsilon} \sum_{r_{1}, \cdots, r_{p+q}=0}^{m-1} c_{0}^{2} c^{p+q} \int_{\delta}^{1} \ldots \int_{0}^{1}\left|\left\langle\sum_{i=1}^{p+q} r_{r_{i}}\left(y_{i}\right)\right\rangle\right| \sum_{i=1}^{p+q} d y_{i}
\end{aligned}
$$

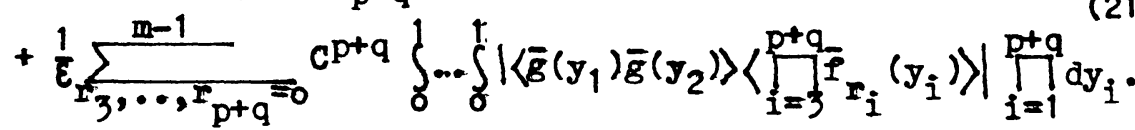

Let $h(y, w)$ be a weakIy correlated process with the correlation Iength $\varepsilon,|h(y, w)|<\eta$ almost everywhere and $I \geq 2$. Then wo estimate 
an integral of the form

For $I^{2} \geq 1 /(2 \varepsilon)^{0}$ we have

$$
\left.\int_{0}^{1} \ldots \int_{0}^{1} k n\left(y_{1}\right) \ldots h\left(y_{I}\right)\right\rangle \mid d y_{1} \ldots d y_{I} \text {. }
$$

$$
\int_{0}^{1} \ldots \int_{0_{2}}^{1}\left\langle h\left(y_{1}\right) \ldots h\left(y_{I}\right)\right\rangle \mid d y_{1} \ldots d y_{I} \leqslant \eta^{I} \leqslant 2 e I^{2} \eta^{I}
$$

and for $I^{2}<1 /(2 c)$

$$
\left.\int_{0}^{1} \ldots \int_{0}^{1} k h\left(y_{1}\right) \ldots h\left(y_{I}\right)\right\rangle \mid d y_{1} \ldots d y_{I} \leqslant \eta^{I}\left(1-v_{I}\right) \text {. }
$$

$\nabla_{1}$ designes the volume of the points $\left(y_{1}, \ldots, y_{I}\right)$ of $[0,1]^{I}$ for which the $\varepsilon$-maximum adjoining splitting is $\left\{\left(y_{1}\right),\left(y_{2}\right), \ldots,\left(y_{1}\right)\right\}$. Since

$$
\nabla_{I}=\int_{0}^{1} d y_{1} \underset{\left|y_{1}-y_{2}\right|>\varepsilon}{\int d y_{2}} \cdots \int_{\left|y_{1}-y_{I}\right|>\varepsilon} d y_{1} \geq \prod_{t=1}^{I-1}(1-2 \varepsilon t) \geq 1-2 \varepsilon I^{2}
$$

and we obtain for aII $I \geq 2$

$$
\left|y_{I-1}: y_{1}\right|>\varepsilon
$$

$$
\int_{0}^{1} \ldots \int_{0}^{1}\left|\left\langle h\left(y_{1}\right) \ldots h\left(y_{I}\right)\right\rangle\right| d y_{1} \ldots d y_{I} \leq 2 \varepsilon I^{2} \eta^{I} \text {. }
$$

Under the condition $N_{0} \geq \frac{m}{2}+3$ a factor of the form as in (22) with $h(x, \omega) \in\left\{\bar{f}_{0}(x, \omega), \ldots, \bar{f}_{m-1}(x, \omega)\right\}$ is included in every item of $(21)$ because the processes $\bar{f}_{r}(x, \omega), \bar{g}(x, \omega)$ are independent. Hence it follows form (21) $\left|\frac{1}{\varepsilon}\left\langle u_{p}(x) u_{q}(x)\right\rangle\right| \leqslant 2(m \eta c)^{p+q-2} c^{2}(p+q)^{2}\left[m^{2} c_{o}^{2} \eta^{2}+\int_{o O}^{1} \int_{0}^{1}\left|\left\langle\bar{g}\left(y_{1}\right) \bar{g}\left(y_{2}\right)\right\rangle\right| d y_{1} d y_{2}\right.$. The uniform convergence from (20) we obtain through the convergence of the series $\sum_{p, q=1}^{\infty}(p+q)^{2}(m \eta c)^{p+q}$ for $|m \eta c|<1$. The theorem 9 is proved.

REMARK 2. Let $\bar{f}_{r}(x, w) \equiv 0$ for $r=0,1, \ldots, m-1$, i.e. Iet $L$ a deterministic operator, then the statement of theorem 9 is right without someone ristriction of the weakly correlated process $\bar{g}(x, \omega)$ related to the smallness. This follows from the proof of theorem 9 or through a direct use of the theorems 1 and 3 on the solution of this boundary value problem

$$
u(x, w)=u_{0}(x)+\int_{0}^{1} G(x, y) \bar{g}(y, w) d y \quad \text { with } u_{0}(x)=\int_{0}^{1} G(x, y)\langle g(y)\rangle d y \text {. }
$$


Hence we obtain in this case that $\frac{1}{\sqrt{\varepsilon}}\left(u(x, \omega)-u_{0}(x)\right)$ converges in the distribution to a Gaussian process $\eta(x, w)$ with

$$
\langle\eta(x)\rangle=0,\langle\eta(x) \eta(y)\rangle=\int_{0}^{1} a_{g}(z) G(x, z) G(y, z) d z .
$$

W.E. Boyce deals with this case of a stochastic boundary value probIem in [1] and he showed that $\frac{1}{\sqrt{\varepsilon}}\left(u(x, \omega)-u_{0}(x)\right)$ is in the Iimit a Gaussian random variable $\eta(x)$ for any $x \in[0,1]$.

REMARK 3. Let $w_{i}(x)$ be the eigenfunctions of the averaged operator $\langle I\rangle$ with $\int_{0}^{1} w_{i}(x) w_{j}(x) d x=\delta_{i j}$ and $\mu_{i}$ the eigenvalues of $\langle I\rangle$. Then we can calculate the correlation function of the limit process $\eta(x, w)$ of $\frac{1}{\sqrt{\varepsilon}}\left(u(x, w)-u_{0}(x)\right)$ through

with

$$
\langle\eta(x) \eta(y)\rangle=\sum_{p, q=1}^{\infty}\left\{b_{p q}+\sum_{r=0}^{m-1} c_{p q}^{r}\right\} \frac{w_{p}(x) w_{q}(y)}{\mu_{p} \mu_{q}}
$$

$$
\begin{aligned}
& b_{p q} \doteq \int_{0}^{1} a_{g}(z) w_{p}(z) w_{q}(z) d z \text { and } \\
& c_{p q}^{r} \doteq \int_{0}^{1} a_{r}(z)\left(u_{0}^{(r)}(z)\right)^{2} w_{p}^{(r)}(z) w_{q}^{(r)}(z) d z .
\end{aligned}
$$

Specially we have for a wide-sense stationary, weakly correlated process

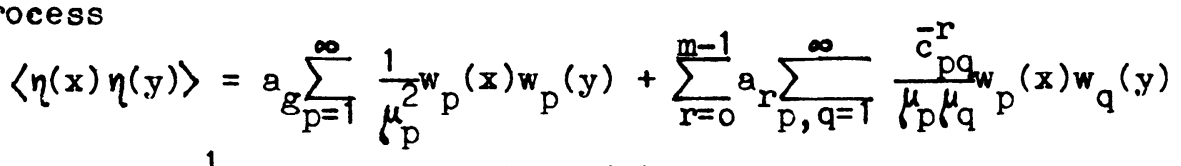

with $\bar{c}_{p q}^{r} \doteq \int_{0}^{1}\left(u_{0}^{(r)}(z)\right)^{2} w_{p}^{(r)}(z) w_{q}^{(r)}(z) d z$.

These statements can be proved easly with $G(x, y)=\sum_{p=1}^{\infty} \frac{w_{p}(x) w_{p}(y)}{\mu_{p}}$ (see also [7]).

Now we regard the boundary value problem (15) with the conditions given above. It denotes $\left\{\varphi_{i}\right\}_{1 \leqslant i<\infty}$ a system of functions of the energetic space $\left.\mathrm{H}_{\langle L\rangle}\right\rangle^{\bullet}$ This system be complete in $\mathrm{H}_{\langle L\rangle}$. The solution $u(x, w)$ of the equation $L(\omega) u=g(\omega)$ is the minimum of the energetic functional $(\mathrm{Lu}, \mathrm{u})-2(\mathrm{~g}, \mathrm{u})$ in the energetic space. The Ritz-method with the co-ordinate functions $\left\{\varphi_{i}\right\}$ conducts to the following system 


$$
\sum_{k=1}^{n}\left(L(\omega) \varphi_{k}, \varphi_{j}\right) x_{k}^{(n)}=\left(g, \varphi_{j}\right), \quad j=1,2, \ldots, n \text {. }
$$

It is $u_{n}(x, w)=\sum_{k=1}^{n} x_{k}^{(n)}(w) \varphi_{k}(x)$ the n-th Ritz-approximation of the solution $u(x, w)$ of $L(w) u=g(w)$. We can write the formula (23) in the following form:

$$
\left(\underline{A}_{0}+\underline{B}^{(\omega))} \underline{\underline{x}}^{(n)}=\underline{b}_{0}+\underline{c}(\omega)\right.
$$

with $\underline{A}_{0}=\left(\left(\langle L\rangle \varphi_{k}, \varphi_{j}\right)\right)_{1 \leqslant k, j \leqslant n}$,

$$
\begin{array}{ll}
\underline{A}_{0}=\left(\left(\langle L\rangle \varphi_{k}, \varphi_{j}\right)\right)_{1 \leqslant k, j \leqslant n}, & \underline{b}_{0}=\left(\left(\langle g\rangle, \varphi_{j}\right)\right)_{1 \leqslant j \leqslant n}^{T}, \\
\underline{B}(\omega)=\left(\left(L_{1}(\omega) \varphi_{k}, \varphi_{j}\right)\right)_{1 \leqslant k, j \leqslant n^{\prime}}, & \underline{c}(\omega)=\left(\left(\bar{g}, \varphi_{j}\right)\right)_{1 \leqslant j \leqslant n}^{\mathrm{T}}
\end{array}
$$

The matrix $A_{0}$ is regular through the conditions for $\left\{\varphi_{j}\right\}$ : $\underline{A}_{0}^{-1}=\left(\alpha_{i j}\right)_{1 \leqslant i, j \notin n}$. Let $\underline{x}_{0}^{(n)}=\underline{A}_{0}^{-1} \underline{b}_{0}=\left(x_{01}^{(n)}, \ldots, x_{o n}^{(n)}\right)^{T}$ be then we obtain for the $n$-th Ritz-approximation $u_{n}(x, \omega)$

$$
\begin{aligned}
u_{n}(x, \omega)=\sum_{k=1}^{n} & x_{o k}^{(n)} \varphi_{k}(x)+\sum_{k=1}^{n}\left(\underline{A}_{0}^{-1}\left(-\underline{B x}_{0}^{(n)}+\underline{c}\right)\right)_{k} \varphi_{k}(x) \\
& + \text { terms of higher order than first in } b_{i j}, c_{j}
\end{aligned}
$$

respectively with $u_{o n}(x) \doteq \sum_{k=1}^{n} x_{o k}^{(n)} \varphi_{k}(x)$

$$
\begin{aligned}
u_{n}(x, w)-u_{o n}(x) & =\left(\bar{g}(z), \sum_{k, j=1}^{n} \alpha_{k j} \varphi_{j}(z) \varphi_{k}(x)\right)_{z} \\
& -\sum_{r=0}^{m-1}\left(\bar{f}_{r}(z), \sum_{k=j=1}^{n} \alpha_{k j} \varphi_{j}^{(r)}(z) \varphi_{k}(x) \sum_{1=1}^{n} x_{o l}^{(n)} \varphi_{1}^{(r)}(z)\right)_{z}
\end{aligned}
$$

+ terms of higher order than first in $b_{i j}, c_{j}$

$\left.\left(\left(h_{1}(z, x), h_{2}(z, x)\right)\right)_{z} \dot{=} \int_{0}^{1} h_{1}(z, x) h_{2}(z, x) d z\right)$. From theorem 5 follows that $\frac{1}{\sqrt{\varepsilon}}\left(u_{n}(x, w)-u_{\text {on }}(x)\right)$ converges in the distribution as $\varepsilon+0$ to the Gaussian process $\eta_{n}(x, \omega)$ with $\left\langle\eta_{n}(x)\right\rangle=0$ and

$$
\begin{aligned}
& \left\langle\eta_{n}(x) \eta_{n}(y)\right\rangle=\sum_{k, j, p, q=1}^{n} \alpha_{k j} \alpha_{p q} \varphi_{k}(x) \varphi_{p}(y)\left(a_{g} \varphi_{j}, \varphi_{q}\right) \\
& +\sum_{r=0 k, j, p, q=1}^{m-1} \sum_{1, j=1}^{n} \alpha_{k j} \alpha_{p q} x_{o l}^{(n)} x_{o i}^{(n)} \varphi_{k}(x) \varphi_{p}(y)\left(a_{r} \varphi_{j}^{(r)} \varphi_{q}^{(r)}, \varphi_{I}^{(r)} \varphi_{i}^{(r)}\right) .
\end{aligned}
$$

Particularly we obtain for $\varphi_{i}(x)=w_{i}(x)$ (see remark 3 ) and wide-sense stationary, weakly correlated processes

$$
\begin{aligned}
\left\langle\eta_{n}(x) \eta_{n}(y)\right\rangle & =a_{g} \sum_{k=1}^{n} \frac{1}{\mu_{k}^{2} w_{k}(x) w_{k}(y)+} \\
& +\sum_{r=0}^{m-1} a_{r_{k}} \sum_{1=1}^{n} \frac{1}{\mu_{k} \mu_{l}}\left(\left(u_{o n}^{(r)}\right)^{2}, w_{k}^{(r)} w_{l}^{(r)}\right) w_{k}(\dot{x}) w_{1}(y)
\end{aligned}
$$


an approximation of the correlation function how it has been represented in the remark 3. The formula is used by an approximation of the correlation function $\langle\eta(x) \eta(y)\rangle$ and (24) is suitable for a explicit calculation of $\langle\eta(x) \eta(y)\rangle$ if the Green function is calculable difficult.

4.2. EXAMPLE. Let $g(x, w)$ be a wide-sense stationary, weakly correlated process then the simple boundary value problem

$$
-u^{n}+b u=g(x, w), \quad u(0)=u(1)=0
$$

with b=const. possesses the averaged problem

$$
-w^{n}+b w=0, \quad w(0)=w(1)=0 .
$$

This problem has only the trivial solution when $b \neq-(k \pi)^{2}$ is and the Green function $G_{b}(x, y)$ is

$$
G_{b}(x, y)=\left\{\begin{array}{ll}
\frac{\operatorname{sh}(\beta x) \operatorname{sh}(\beta(1-y))}{\operatorname{sh}(\beta)} & \text { for } 0 \leq x<y \leq 1 \\
\frac{\operatorname{sh}(\beta y) \operatorname{sh}(\beta(1-x))}{\operatorname{sh}(\beta)} & \text { for } 0 \leq y<x \leq 1
\end{array} \quad(\beta=\sqrt{b}) .\right.
$$

Hence we obtain the correlation function for the limit process $\eta_{b}(x, w)$ from theorem 9

and the variance

$$
\left\langle\eta_{b}(x) \eta_{b}(y)\right\rangle=a \int_{0}^{1} G_{b}(x, z) G_{b}(y, z) d z
$$

$$
\left\langle\eta_{b}^{2}(x)\right\rangle=a \int_{0}^{1} G_{b}^{2}(x, z) d z=\frac{a}{\beta^{2} \operatorname{sh}^{2}(\beta)}\left(f_{\beta}(x)+f_{\beta}(1-x)\right)
$$

with $f_{\beta}(x)=\operatorname{sh}^{2}(\beta(1-x))\left(\frac{1}{4 \beta} \operatorname{sh}(2 \beta x)-\frac{1}{2} x\right)$. a is the parameter which belongs to the wide-sense stationary, weakly correlated process $g(x, \omega)$. It is $\beta=i \sqrt{-b}$ for $b<0$ and

$$
\left\langle\eta_{b}^{2}(x)\right\rangle=\frac{a}{\bar{\beta}^{2} \sin ^{2}(\bar{\beta})}\left(\bar{f}_{\bar{\beta}}(x)+\bar{f}_{\bar{\beta}}(1-x)\right)
$$

with $\bar{f}_{\bar{\beta}}(x)=-\sin (\bar{\beta}(1-x))\left(\frac{1}{4 \bar{\beta}^{\prime}} \sin (2 \bar{\beta} x)-\frac{1}{2} x\right)$. Expecially we have

$$
\lim _{b \rightarrow 0}\left\langle\eta_{b}^{2}(x)\right\rangle=\frac{1}{3} x^{2}(1-x)^{2} \text { and } \operatorname{Iim}_{b \rightarrow-(k x)^{2}}\left\langle\eta_{b}^{2}(x)\right\rangle=\infty \text {. }
$$

In the following Fig. 4 the variance of the limit process $\eta_{b}(x, \omega)$ is 
plotted for some values of the parameter b. Since $\left\langle\eta_{b}^{2}(x)=\left\langle\eta_{b}^{2}(1-x)\right\rangle\right.$, we have plotted this function for $0 \leqslant x \leqslant \frac{1}{2}$ only. The following values submit for $b=\pi^{2}-\frac{1}{2}$ by contrast to it:

\begin{tabular}{|c|c|c|c|c|c|c|}
\hline $\mathbf{x}$ & 0 & 0,1 & 0,2 & 0,3 & 0,4 & 0,5 \\
\hline$\frac{1}{a}\left\langle\eta_{b}^{2}(x)\right\rangle$ & 0 & 0,7650 & 2,7663 & 5,2382 & $.7,2570$ & 8,0004 \\
\hline
\end{tabular}

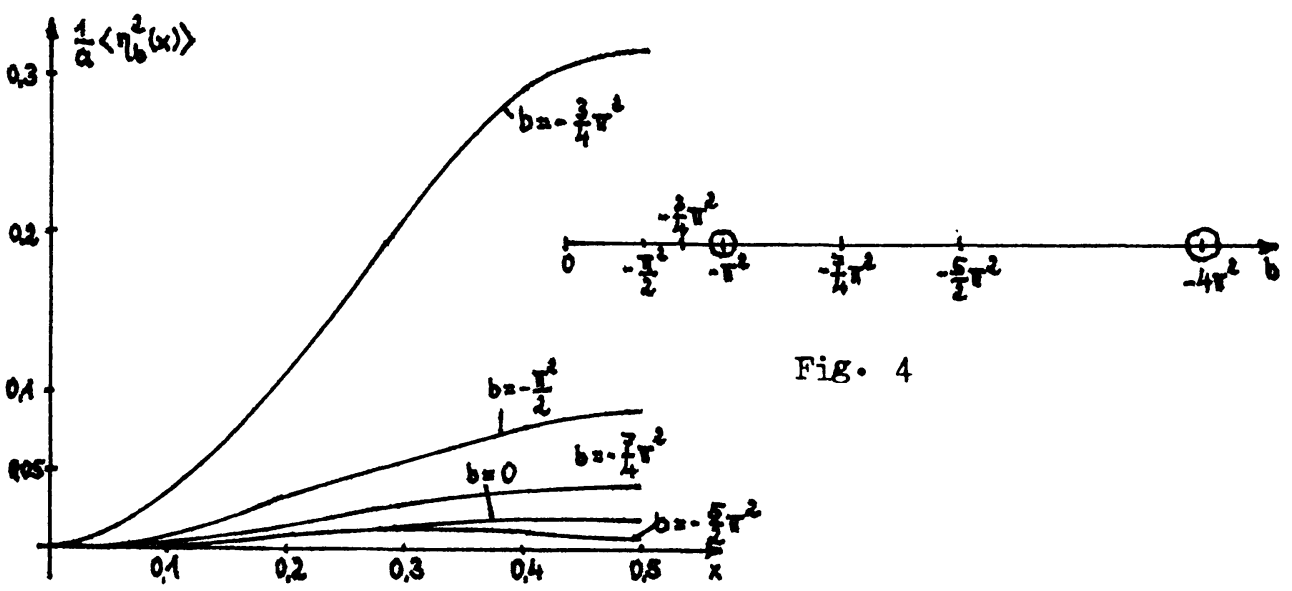

5. STOCHASTIC INITIAL VALUE PROBLEMS

We consider a system of ordinary differential equations of the first order

$$
\frac{d \underline{x}}{d \bar{t}}=\underline{A}(t) \underline{x}+\underline{f}(t, \omega)
$$

with the initial condition $\underline{\underline{x}}\left(t_{0}\right)=\underline{x}_{0} \cdot \underline{A}(t)$ is a $n \times n$ deterministic matrix, $\underline{\Delta}(t)=\left(a_{i j}(t)\right)_{1 \leqslant i, j \notin n}$, $\underline{x}$ is the vector $\underline{x}(t)=\left(x_{i}(t)\right)_{1 \leqslant i \leqslant n}^{T}$, $\underline{x}_{0}=\left(\underline{x}_{i}\right)_{1 \leqslant i \leqslant n}^{T}$ and $\underline{f}(t, \omega)=\left(f_{i}(t, w)\right)_{1 \leqslant i \leqslant n}^{T}$ is a stochastic vector process. Let $f_{i}(t, \omega)$ be procesees of which a.s. the trajectories are continuous and $\underline{Q}\left(t, t_{0}\right)$ be the principal matrix associated with $\underline{A}(t)$ (i.e. $\underline{Q}\left(t_{0}, t_{0}\right)=\underline{I}$ ( $\underline{I}$ is the identity matrix) and $\frac{d}{d t} \underline{Q}\left(t, t_{0}\right)=$ $\left.\underline{A}(t) \underline{Q}\left(t, t_{0}\right)\right)$, then the unique solution $a_{. s}$. of the initial value problem (26) may be written in the form 


$$
\underline{\boldsymbol{x}}(t, \omega)=\underline{Q}\left(t, t_{0}\right)\left(\underline{x}_{0}+\int_{t_{0}}^{t} \underline{Q}^{-1}\left(s, t_{0}\right) \underline{f}(s, \omega) d s\right) .
$$

The integral is defined by the integral of sample functions. In generally we cannot calculate the distribution of the solution $\underline{x}(t, \omega)$ if we know the distribution of $\underline{f}(t, \omega)$. Let $\underline{f}(t, \omega)$ be a Gaussian vector process then is $\int_{t_{0}}^{t} \underline{Q}^{-1}\left(s, t_{0}\right) \underline{f}(s, \omega)$ ds also a Gaussian vector process and in the same way the solution $\underline{x}(t, \omega)$ of this system of linear differential equations (see $/ 2 /$ ). The first moments of the solution are

$$
\begin{aligned}
& \langle\underline{x}(t)\rangle=\underline{Q}\left(t, t_{0}\right)\left(\underline{x}_{0}+\int_{t_{0}}^{t} \underline{Q}^{-1}\left(s, t_{0}\right)\langle\underline{f}(s)\rangle d s\right) \quad \text { and } \\
& \left.\left.\underline{R}\left(t_{1}, t_{2}\right) \doteq \underline{\underline{x}}\left(t_{1}\right)-\left\langle\underline{x}\left(t_{1}\right)\right\rangle\right]\left[\underline{x}\left(t_{2}\right)-\left\langle\underline{x}\left(t_{2}\right)\right\rangle\right]^{T}\right\rangle \\
& =\underline{Q}\left(t_{1}, t_{0}\right) \int_{t_{0}}^{t} \underline{Q}^{-1}\left(s_{1}, t_{0}\right) \underline{R}\left(s_{1}, s_{2}\right) \underline{Q}^{-T}\left(s_{2}, t_{0}\right) d s_{1} d s_{2} \underline{Q}^{T}\left(t_{2}, t_{0}\right)
\end{aligned}
$$

with

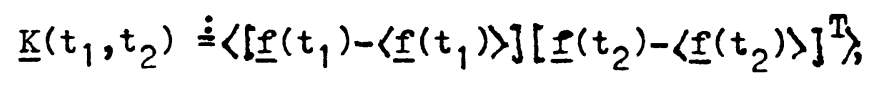

provided that

$$
\int_{t_{0}}^{t_{1}}\langle\|\underline{e}(s)\|\rangle d s<\omega, \quad \int_{t_{0}}^{t_{1}} \int_{0}^{t}\left\langle\left\|\underline{f}\left(s_{1}\right) \underline{e}^{T}\left(s_{2}\right)\right\|\right\rangle d s_{1} d s_{2}<\infty
$$

for all $t_{1}, t_{2} \geq t_{0}$ ( $\|$. $\|$ denotes the Euklidian norm of $R^{n}$ ).

In the following we consider the solution $(26)$ if $\underline{f}(t, \omega)=$ $\frac{1}{\sqrt{\varepsilon}} g_{\varepsilon}(t, \omega)$ denotes a vector process with independent, weakly correlated processes $g_{i \varepsilon}(t, w)$ as components. This leads to the following theorem.

THEOREM 10. Let $g_{\varepsilon}(t, w)$ a sequence of weakly correlated vector processes for $\varepsilon \nmid O$ with independent components $g_{i \varepsilon}(t, \omega)$ and $\left\langle g_{i \varepsilon}(t) g_{i \varepsilon}(s)\right\rangle=\left\{\begin{array}{cl}R_{i \varepsilon}(t, s) & \text { for } s \in K_{\varepsilon}(t) \\ 0 & \text { for } s \notin K_{\varepsilon}(t)\end{array}, \lim \frac{1}{\varepsilon} \int_{-\varepsilon}^{\varepsilon} R_{i \varepsilon}(t, t+s) d s=a_{i}(t)\right.$, then the solution

with

$$
\underline{x}_{\varepsilon}(t, w)=\underline{y}(t)+\frac{1}{\sqrt{\varepsilon}} Q\left(t, t_{0}\right) \int_{t_{0}}^{t} \underline{Q}^{-1}\left(s, t_{0}\right)_{g_{\varepsilon}}(s, w) d s
$$


of the initial value problem

$$
\underline{Z}(t)=\underline{Q}\left(t, t_{0}\right)\left(\underline{x}_{0}+\int_{t_{0}}^{t} \underline{Q}^{-1}\left(s, t_{0}\right) \underline{h}(s) d s\right)
$$

$$
\frac{d \bar{x}}{d \bar{t}}=\underline{A}(t) \underline{x}+\underline{h}(t)+\frac{1}{\sqrt{\varepsilon}} \underline{G}_{\varepsilon}(t, \omega), \quad \underline{x}\left(t_{0}, \omega\right)=\underline{x}_{0}
$$

converges in the distribution to a Gaussian vector process

$$
\eta(t, w)=y(t)+\underline{y}(t, w)
$$

with $\langle\mathbf{p}(t)\rangle=0$ and

$\left\langle\mathbf{s}\left(t_{1}\right)\left(t_{2}\right)^{\mathrm{T}}\right\rangle=\sum_{k=1}^{n} \int_{t_{0}}^{\min \left(t_{1}, t_{2}\right)}\left(a_{k}(s) q_{i k}\left(t_{1}, t_{0} ; s\right) q_{j k}\left(t_{2}, t_{0} ; s\right)\right)_{1 \leqslant i, j k n} d s$. It is $\left.\left(a_{i j}\left(t, t_{0} ; s\right)\right)\right)_{1 \leqslant i, j \leqslant n} \stackrel{\dot{=}}{Q}\left(t, t_{0}\right) \underline{Q}^{-1}\left(s, t_{0}\right)$.

PROOF. The proof is following from theorem 5 then we obtain with the definition of the $q_{i j}$

$$
\underline{x}_{\varepsilon}(t, \omega)=z(t)+\frac{1}{\sqrt{\varepsilon}} \sum_{k=1}^{n} \int_{t_{0}}^{t^{i j}}\left(q_{i k}\left(t, t_{0} ; s\right) g_{k \varepsilon}(s, \omega)\right)_{1 \leqslant i \leqslant n} d s
$$

and from this for the limit process $(t, \omega)=\left(J_{i}(t, \omega)\right)_{16 i \text { in }}$ with the notations of theorem 5

$$
\begin{aligned}
\left\langle\xi_{i}\left(t_{1}\right) \xi_{j}\left(t_{2}\right)\right\rangle & =\sum_{k=1}^{n}\left\langle\xi_{i k}\left(t_{1}\right) \xi_{j k}\left(t_{2}\right)\right\rangle \\
& =\sum_{k=1}^{n} \min \left(t_{1}, t_{2}\right) a_{0}(s) q_{i k}\left(t_{1}, t_{0} ; s\right) q_{j k}\left(t_{2}, t_{0} ; s\right) d s .
\end{aligned}
$$

In generally $(t, \omega)$ does not denote a wide-sense stationary vector process while $\left\langle\left(t_{1}\right) t^{T}\left(t_{2}\right)\right\rangle$ is not a function which only depends on $t_{2}-t_{1}$. We assume that the processes $g_{i \varepsilon}(t, \omega)$ are wide-sense stationary and weakly correlated and $\underline{A}(t)=\underline{A}$ is a constant matrix, the eigenvalues $\lambda_{j}$ of which have negative real parts. Then we obtain

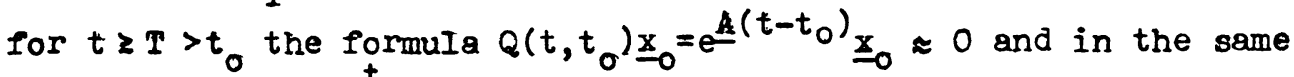
way the solutions $\int_{t_{0}}^{t} e^{A(t-s)}\left(\underline{h}(s)+\frac{1}{\sqrt{E}} g_{\varepsilon}(s)\right) d s$ and $\int_{-\infty}^{t} e^{A(t-s)}\left(\underline{h}(s)+\frac{1}{\sqrt{\varepsilon}} \underline{t}_{\varepsilon}(\varepsilon)\right)$ ds of the system of differential equations differ by a solution of the homogeneous system of (27) which is near. by zero for $t \geq T>t_{0}$. Hence the solution of (27) is described by

$$
\underline{\underline{x}}_{\varepsilon}(t, \omega)=\int_{-\infty}^{t} e^{A(t-s)} \underline{h}(s) d s+\frac{1}{\sqrt{\varepsilon}} \int_{-\infty}^{t} e^{A(t-s)} g_{\varepsilon}(s, w) d s .
$$


Then the term $\frac{1}{\sqrt{\varepsilon}} \int_{-\infty}^{t} e^{A(t-s)} g_{\varepsilon}(s, \omega) d s$ is wide-sense stationary as we obtain from

$$
\left\langle\int_{-\infty}^{t} e^{A(t-s)} g_{\varepsilon}(s, w) d s\right\rangle=0
$$

anđ

$$
\begin{aligned}
\left\langle\int_{-\infty}^{t_{1}} e^{A\left(t_{1}-s\right)} g_{\varepsilon}(s, w) d s\left(\int_{-\infty}^{t_{2}} e^{A\left(t_{2}-s\right)} g_{\varepsilon}(s, w) d s\right)^{T}\right\rangle \\
=\int_{-\infty}^{t_{1}} \int_{-\infty}^{t_{2}} e^{A\left(t_{1}-s_{1}\right)}\left\langle g_{\varepsilon}\left(s_{1}\right) g_{\varepsilon}\left(s_{2}\right)^{T}\right\rangle e^{A^{T}\left(t_{2}-s_{2}\right)} d s_{1} d s_{2} \\
=\int_{0}^{\infty} \int_{0}^{\infty} e^{A s 1}\left\langle g_{\varepsilon}\left(t_{1}-s_{1}\right) g_{\varepsilon}\left(t_{2}-s_{2}\right)^{T}\right\rangle e^{A^{T} s_{2}} d s_{1} d s_{2}
\end{aligned}
$$

The theorem 11 can be proved like the theorem 10.

THEOREW 11. Let $g_{\varepsilon}(t, w)$ be a sequence of wide-sense stationary, weakIy correlated vector processes for $\varepsilon \downarrow 0$ with independent components and

$$
\begin{aligned}
& \left\langle g_{i \varepsilon}(t) g_{i \varepsilon}(s)\right\rangle=\left\{\begin{array}{cc}
\sigma_{2 i} \rho_{i \varepsilon}(t-s) & \text { for }|t-s| \leq \varepsilon \\
0 & \text { for }|t-s|>\varepsilon \text {, } \lim \frac{1}{\varepsilon} \int_{-\varepsilon}^{\varepsilon} \rho_{i \varepsilon}(s) d s=a_{i}<\infty
\end{array}\right. \\
& \left(a_{i} \neq 0\right), \text { then the solution } \\
& \underline{\underline{x}}_{\varepsilon}(t, \omega)=\int_{-\infty}^{t} e^{A(t-s)} \underline{h}(s) d s+\frac{1}{\sqrt{\varepsilon}} \int_{-\infty}^{t} e^{A(t-s)} g_{\varepsilon}(s, w) d s
\end{aligned}
$$

of the system of differential equations

$$
\frac{d \underline{x}}{d \bar{t}}=\underline{A x}+\underline{h}(t)+\frac{1}{\sqrt{\varepsilon}} g_{\varepsilon}(t, \omega)
$$

converges in the distribution to a Gaussian vector process

$$
\bar{\eta}(t, \omega)=\int_{-\infty}^{t} e^{A(t-s)} \underline{h}(s) d s+\underline{\Gamma}(t, \omega) .
$$

$\underline{J}(t, \omega)$ is a wide-sense stationary, weakly correlated vector process with $\langle\underline{s}(t)\rangle=0$ and

$$
\left\langle\underline{\underline{I}}\left(t_{1}\right) \underline{\underline{I}}\left(t_{2}\right)^{\mathrm{T}}\right\rangle=\left(\sum_{k=1}^{n} a_{k} \sigma_{2 k} \int_{-\infty}^{\min \left(t_{1}, t_{2}\right)}\left(e^{A\left(t_{1}-s\right)}\right)_{i k}\left(e^{A\left(t_{2}-s\right)}\right)_{j k} d s\right)_{\substack{1 \leqslant i, j \\ k n !}}
$$

Indeed $\underline{J}(t, \omega)$ is a wide-sense stationary vector process because we obtain for $t_{1} \leqslant t_{2}$ $\int_{-\infty}^{\min \left(t_{1}, t_{2}\right)}\left(e^{A\left(t_{1}-s\right)}\right)_{i k}\left(e^{A\left(t_{2}-s\right)}\right) j_{j k} d s=\int_{0}^{\infty}\left(e^{A s}\right)_{i k}\left(e^{A\left(t_{2}-t_{1}+s\right)}\right) j k d s$.

In the following we describe the connection with the Ito differential equations. The Itó differential equation 


$$
d \underline{x}_{t}=\left(\underline{A}(t) \underline{x}_{t}+\underline{h}(t)\right) d t+\underline{G}(t) d \underline{W}_{t}, \quad \underline{x}_{t}(\omega)=\underline{x}_{0}
$$

corresponds to the initial value problem (27) as $\varepsilon \downarrow 0$ where $G(t)=\left(\delta_{i j} \sqrt{a_{i}(t)}\right)_{1 \leqslant i, j \leqslant n}$ and $\underline{W}_{t}=\left(w_{t}^{i}\right)_{1 \leqslant i \leqslant n}$ is the wiener process with independent components. This statement is following from the correspondence of the integral equation

$$
\underline{\underline{x}}(t)=\underline{x}_{0}+\int_{t_{0}}^{t}(\underline{A}(s) \underline{x}(s)+\underline{h}(s)) d s+\frac{1}{\sqrt{\varepsilon}} \int_{t_{0}}^{t} g_{\varepsilon}(s, w) d s
$$

with (27) and of the Ito integral equation

$$
\underline{x}_{t}(w)=\underline{x}_{0}+\int_{t}^{t}\left(\underline{A}(s) \underline{x}_{s}(w)+\underline{h}(s)\right) d s+\int_{t_{0}}^{t} \underline{G}(s) d \underline{W}
$$

with (28) if we take into consideration the convergence in the distribution from theorem 5

We note the relation

$$
\lim _{\varepsilon+0} \frac{1}{\sqrt{\varepsilon}} \int_{t_{0}}^{t} g_{\varepsilon}(s, \omega) \mathrm{d} s=\int_{t_{0}}^{t} \underline{G}(s) \mathrm{dW}_{s} \text {. }
$$

$$
\left.\left\langle\int_{t_{0}}^{t_{1}} \underline{G}(s) d \underline{W}_{s}\left\langle\int_{t_{0}}^{t_{2}} \underline{G}(s) d W_{s}\right)^{T}\right\rangle=\min _{t_{0}} t_{1}, t_{2}\right) \underline{G}(s) \underline{G}(s)^{T} d s
$$

for the Its integral $\int_{t}^{t} G(s) d W_{8}$. It is following from the theory of the Ito differential equations that the solution of (28) (the solution of the integral equation (28)) is a Gaussian vector process with $\left\langle\underline{\underline{x}}_{t}\right\rangle=\underline{Q}\left(t, t_{0}\right)\left(\underline{x}_{0}+\int_{t_{0}}^{t} \underline{Q}^{-1}\left(s, t_{0}\right) \underline{h}(s) d s\right)$ and $\left\langle\left(\underline{x}_{t_{1}}-\left\langle\underline{x}_{t_{1}}\right\rangle\right)\left(\underline{x}_{t_{2}}-\left\langle\underline{x}_{t_{2}}\right\rangle\right)^{T}\right\rangle=$

$$
=\underline{Q}\left(t_{1}, t_{0}\right) \int_{t_{0}}^{\min \left(t_{1}, t_{2}\right)} \underline{\underline{Q}}^{-1}\left(s, t_{0}\right) \underline{G}(s) \underline{G}(s)^{T} \underline{Q}^{-T}\left(s, t_{0}\right) d s \underline{Q}^{T}\left(t_{2}, t_{0}\right) .
$$

A comparison of the limit solution $\eta(t, \omega)$ from theorem 10 with this solution from (28) shows the correspondence of the solution processes: $\underline{x}_{t}(\omega)=\eta(t, \omega)$. Hence the same solutions of the initial value problem (27) is obtained if one takes up the limit value $\varepsilon r 0$ in the equation and the It $\delta$ differential equation solves which one obtains and if one solves (27) in the sample functions and takes up the limit value $\varepsilon \downarrow 0$ in the solution. 
We can make many applications (e.g. the number of threshold crossings and other) because the limit process $\underline{\eta}(t, \omega)$ or $\bar{\eta}(t, \omega)$, respectively, is a Gaussian process. Hence we obtain approximately a good general view of the behaviour of the solution $\underline{x}_{\varepsilon}(t, w)$ or $\underline{\underline{x}}_{\varepsilon}(t, \omega)$, respective$l y$, if weakly correlated processes come in the differential equation.

\section{REFERENCES}

1. Boyce, W. E. Stochastic nonhomogeneous Sturm-Liouville problem, J.Franklin Inst. 282 (1966) 206-215.

2. Bunke, H. Gewöhnliche Differentialgleichungen mit zufglligen Parametern, Akademi e-Verlag, Berlin, 1972.

3. Farkert, W. and vom Schejdt, J. Zum Mittelungsproblem bei stochastischen Eigenwertaufgaben, Beiträge zur Analysis 10 (1977) 47-61.

4. Purkert, W. and vom Scheidt, J. Zur approximativen Lösung des Míttelungsproblems für die Eigenwerte stochastischer Differentialoperatoren, ZAMM 57 (1977) 515-526.

5. Purkert, W. and vom Scheidt, J. Stochastic eigenvalue problems for differential equations, Reports on mathem. physics, Torun (in print).

6. Purkert, W. and vom Scheidt, J. Ein Grenzverteilungssatz für stochastische Eigenwert probleme, ZAMM (in print).

7. Purkert, W. and vom Scheidt, J. Randwertprobleme mit schwach korrelierten Prozessen als Koeffizienten, Transactions of the 8th Prague Conference on Information Theory, Statistical Decision Functions, Random Processes, Prag (1978) 107-118.

8. Unlenbeck, G. E. and Ornstein, L. S. On the theory of Brownian motion, Physical review 36 (1930) 823-841. 


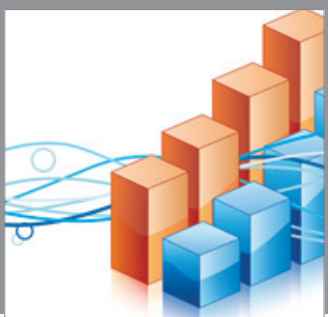

Advances in

Operations Research

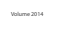

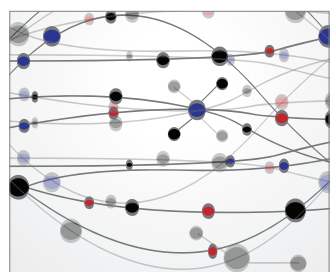

\section{The Scientific} World Journal
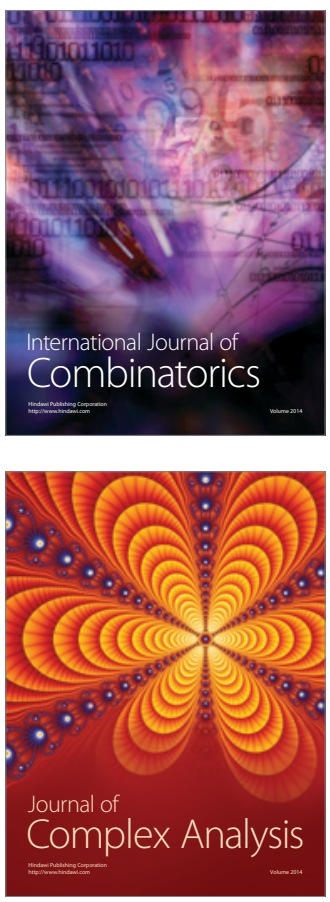

International Journal of

Mathematics and

Mathematical

Sciences
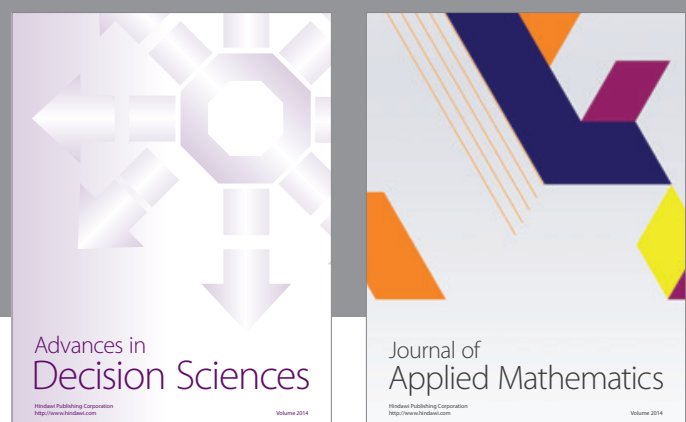

Journal of

Applied Mathematics
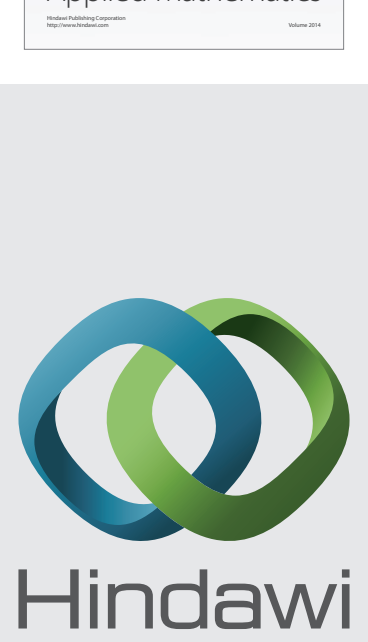

Submit your manuscripts at http://www.hindawi.com
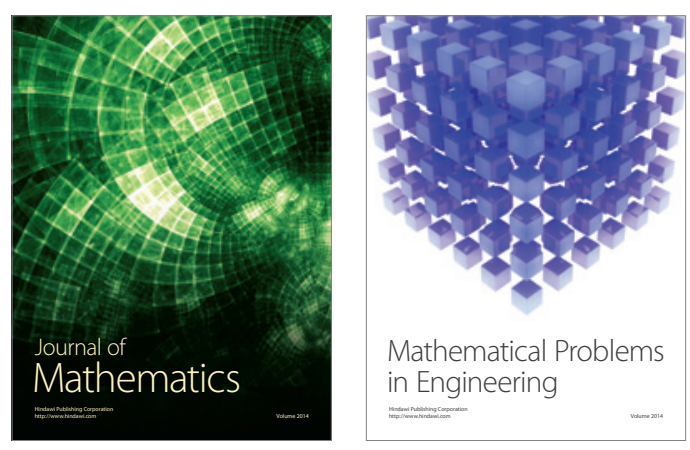

Mathematical Problems in Engineering
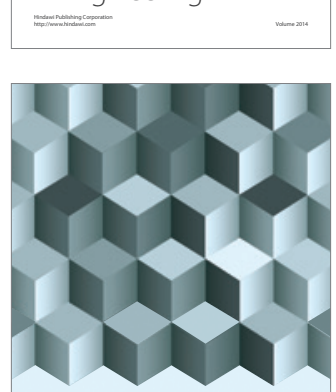

Journal of

Function Spaces
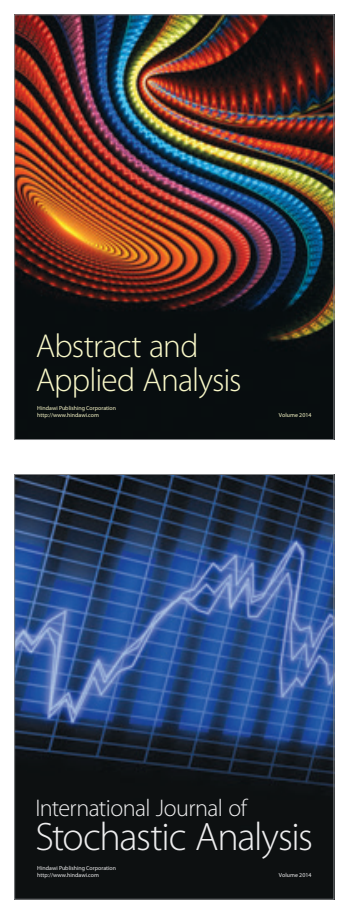

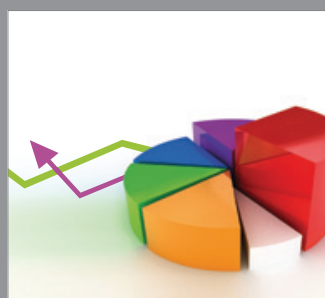

ournal of

Probability and Statistics

Promensencen
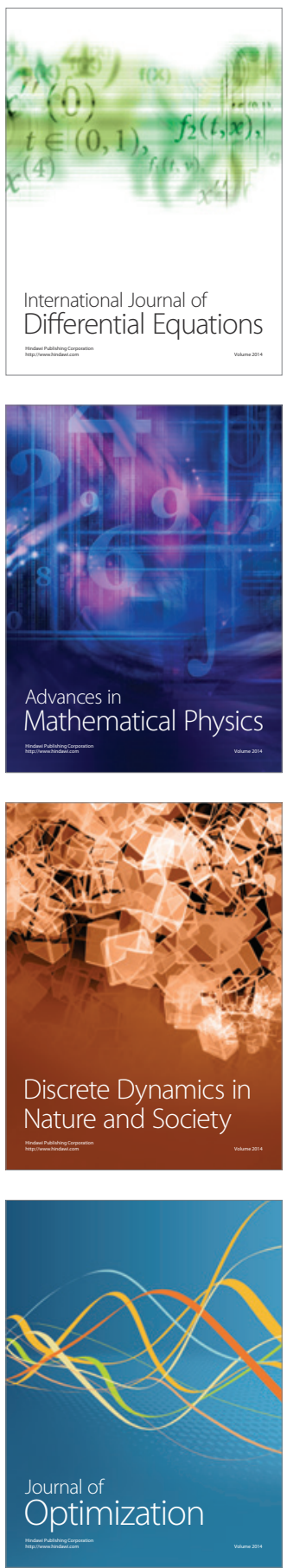